\title{
ON THE TRANSITION GRAPHS OF TURING MACHINES
}

\author{
Didier Caucal * \\ IRISA-CNRS, Campus de Beaulieu, 35042 Rennes, France
}

\begin{abstract}
As for pushdown automata, we consider labelled Turing machines with $\epsilon$-rules. With any Turing machine $M$ and with a rational set $C$ of configurations, we associate the restriction to $C$ of the $\varepsilon$-closure of the transition set of $M$. We get the same family of graphs by using the labelled word rewriting systems. We show that this family is the set of graphs obtained from the binary tree by applying an inverse mapping into $F$ followed by a rational restriction, where $F$ is any family of recursively enumerable languages containing the rational closure of all linear languages. We show also that this family is obtained from the rational graphs by inverse rational mappings. Finally we show that this family is also the set of graphs recognized by (unlabelled) Turing machines with labelled final states, and even if we restrict to deterministic Turing machines.
\end{abstract}

\section{Introduction}

The transition graphs of some classes of machines have already been investigated. First, Muller and Schupp have considered rational restrictions of the transition graphs of pushdown automata: these graphs are the graphs of bounded degree having a finite number of non isomorphic connected components when decomposed by distance from any vertex; these graphs have a decidable monadic theory [MS 85]. This graph family is also the set of rational restrictions of the prefix transition graphs of finite labelled word rewriting systems [Ca 90]. Extending to recognizable labelled rewriting systems, the rational restrictions of their prefix transition graphs define a

* caucal@irisa.fr http://www.irisa.fr/prive/caucal/

Preprint submitted to Elsevier Preprint 
larger family of graphs having a decidable monadic theory [Ca 96]. To extend this last family, Morvan has defined the family of rational graphs, which are the graphs recognized by transducers with labelled final states [Mo 00]. This family is general: it contains for instance the transition graphs of Petri nets, the transition graphs of congruential systems [Ur 00], and the transition graphs of labelled word rewriting systems. Although the rational graphs are recursive, there exists a rational graph with an undecidable first order theory.

There is a simple and uniform way to present all the previous families of graphs from families of languages. Take a family $F$ of languages, a set $T$ of labels and a mapping $h: T \longrightarrow F$ associating to each label a language in $F$. The inverse image $h^{-1}(G)$ of a graph $G$ by $h$ has an arc $s \stackrel{a}{\longrightarrow} t$ when there is a path $s \stackrel{u}{\longrightarrow} t$ in $G$ for some word $u$ in $h(a)$. A family $F$ of languages induces a family $R E C_{F}$ of graphs obtained from the infinite binary tree by marking rationally some vertices (with a special letter) and then applying an inverse mapping. Then the family of rational restrictions of the transition graphs of pushdown automata is the family $R E C_{F i n}$ of graphs induced by the family Fin of finite languages. Furthermore, the family of rational restrictions of the prefix transition graphs of recognizable rewriting systems is the family $R E C_{R a t}$ of graphs induced by the family Rat of rational languages [Ca 96]. Finally, the family of rational graphs is the family $R E C_{\bar{L} \text { in }}$ of graphs induced by a subfamily $\bar{L}$ in of linear languages [Mo 00].

Thus small families of languages induce large families of graphs. Conversely, a family of graphs yields a family of languages. A trace of a graph is the language of path labels from and to given finite vertex sets. The traces of finite graphs are the rational languages. The traces of graphs in $R E C_{F i n}$ are the context-free languages which are also the traces of graphs in $R E C_{\text {Rat }}$. Finally, the traces of rational graphs are the context-sensitive languages [MoS 01].

Following the Chomsky hierarchy, we present a general family of graphs, the traces of which are the recursively enumerable languages. We consider the off-line Turing machines [MS 97] with a read only one way input tape and a unique two ways working tape. These machines are particular labelled word rewriting systems allowing rules labelled by $\varepsilon$. Following [Pay 00] and as for prefix transition graphs of word rewriting systems, we consider rational restrictions of the $\varepsilon$-closure of transition graphs of these off-line Turing machines. We show that this family of graphs coincides with the family of rational restrictions of the $\varepsilon$-closure of the transition graphs of word rewriting systems. We also show that this graph family is equal to $R E C_{\bar{L}_{\text {in }(R a t)}}=R E C_{R E}$ meaning that it is induced by any language family between the rational closure of $\bar{L}$ in and the family $R E$ of recursively enumerable languages. Furthermore, we show that this graph family is obtained by inverse rational mappings of rational graphs. Finally and as for the rational graphs recognized by transducers with labelled final states, we show that our family is the set of graphs recognized by (usual) nondeterministic Turing machines with labelled final states, and this result remains true if we restrict to deterministic Turing machines. 


\section{Preliminaries on graphs}

Let $P$ be a subset of a monoid $M$, and $I d_{P}=\{(u, u) \mid u \in P\}$ the identity relation on $P$. A (simple oriented labelled) $P$-graph $G$ is a subset of $V \times P \times V$ where $V$ is an arbitrary set. Any $(s, a, t)$ of $G$ is a labelled arc of source $s$, of target $t$, with label $a$, and is identified with the labelled transition $s \underset{G}{\rightarrow} t$ or directly $s \stackrel{a}{\longrightarrow} t$ if $G$ is understood. We denote by $V_{G}:=\{s \mid \exists a \exists t, s \stackrel{a}{\longrightarrow} t \vee t \stackrel{a}{\longrightarrow} s\}$ the vertex set of $G$. The set $2^{V \times P^{*} \times V}$ of $P^{*}$-graphs with vertices in $V$ is a monoid for the composition $G \circ H:=\{r \stackrel{a \cdot b}{\longrightarrow} t \mid \exists s, r \underset{G}{\stackrel{a}{\longrightarrow}} s \wedge s \underset{H}{\rightarrow} t\}$ for any $G, H \subseteq V \times P^{*} \times V$, where $\{s \stackrel{1}{\longrightarrow} s \mid s \in V\}$ is its neutral element. The submonoid $\{G\}^{*}$ of $2^{V_{G} \times P^{*} \times V_{G}}$ generated by any graph $G$ gives by union the graph $G^{*}:=\bigcup\{G\}^{*}$. The relation $\underset{G^{*}}{\stackrel{u}{\longrightarrow}}$ denoted by $\underset{G}{\stackrel{u}{\longrightarrow}}$ or simply by $\stackrel{u}{\Longrightarrow}$ if $G$ is understood, is the existence of a path in $G$ labelled $u \in P^{*}$. For every $Q \subseteq P^{*}$, we write $s \stackrel{Q}{\Longrightarrow} t$ if there is some $u \in Q$ such that $s \stackrel{u}{\Longrightarrow} t$. The restriction $G_{\mid C}$ of a $P$-graph $G$ to an arbitrary set $C$ is $G_{\mid C}:=G \cap(C \times P \times C)$. The labels $\mathcal{L}(G, E, F)$ of paths of $G$ from a set $E$ to a set $F$ is the set $\mathcal{L}(G, E, F):=\{u \in M \mid \exists s \in E, \exists t \in F, s \underset{G}{\stackrel{u}{\longrightarrow}} t\}$. A trace of a graph $G$ is the language $\mathcal{L}(G, E, F)$ of path labels from a finite set $E$ to a finite set $F$.

Given an alphabet $T$ and a relation $h \subseteq T \times P$, the inverse $h^{-1}(G)$ by $h$ of any $P$ graph $G$ is the following $T$-graph:

$$
h^{-1}(G):=\{s \stackrel{a}{\longrightarrow} t \mid a \in T \wedge \exists u \in h(a), s \underset{G}{\stackrel{u}{\longrightarrow}} t\}
$$

An example is given Figure 2.3.

A relation $h \subseteq T \times P$ can be seen as a mapping from $T$ into $2^{P}$ associating the image $h(a)$ of any $a \in T$. When $P=S^{*}$ for some alphabet $S$, such a mapping is extended by morphism to a substitution from $T^{*}$ into $S^{*}$ i.e. a mapping $h$ from $T^{*}$ into $2^{S^{*}}$ such that $h(\varepsilon)=\{\varepsilon\}$ and $h(u v)=h(u) h(v)$ for every $u, v \in T^{*}$. The composition of functions is the composition of their relations: $(g \circ h)(a)=h(g(a))$. Let us give basic properties of inverse mappings.

Lemma 2.1 Let $O, P, Q$ be alphabets and $G$ be a $Q$-graph.

Let $h \subseteq P \times Q^{*}$ and $g \subseteq O \times P^{*}$ extended by substitution. We have
a) $s \underset{h^{-1}(G)}{\stackrel{u}{\longrightarrow}} t \Longleftrightarrow s \underset{G}{\stackrel{h(u)}{\longrightarrow}} t$ for any $u \in P^{+}$and $s, t \in V_{G}$
b) $\mathcal{L}\left(h^{-1}(G), E, F\right)=h^{-1}(\mathcal{L}(G, E, F))$ for any $E, F \subseteq V_{h^{-1}(G)}$
c)

$$
\begin{aligned}
g^{-1}\left(h^{-1}(G)\right) & =\left((g \circ h)^{-1}(G)\right)_{\mid V_{h^{-1}(G)}} \\
\text { and } \quad g^{-1}\left(h^{-1}(G)\right) & =(g \circ h)^{-1}(G) \quad \text { if } \varepsilon \notin g(O) .
\end{aligned}
$$

\section{Proof.}


i) We prove (a) by induction on the length of any word $u \in P^{+}$. $u=a \in P: \forall s, t \in V_{G} \quad s \underset{h^{-1}(G)}{\stackrel{a}{\Longrightarrow}} t \Longleftrightarrow s \underset{h^{-1}(G)}{\stackrel{a}{\longrightarrow}} t \Longleftrightarrow s \underset{G}{\stackrel{h(a)}{\rightleftarrows}} t$.

$u=v w$ with $v, w \in P^{+}$: for any $s, t \in V_{G}$, we have

$$
\begin{aligned}
& s \underset{h^{-1}(G)}{\stackrel{v w}{\Longrightarrow}} t \Longleftrightarrow \exists r, s \underset{h^{-1}(G)}{\stackrel{v}{\Longrightarrow}} r \underset{h^{-1}(G)}{\stackrel{w}{\Longrightarrow}} t \\
& \Longleftrightarrow \exists r, s \stackrel{h(v)}{\rightleftharpoons} r \stackrel{h(w)}{\Longrightarrow} t \quad \text { by induction hypothesis } \\
& \Longleftrightarrow s \stackrel{h(v) h(w)}{\Longrightarrow} t \quad G \text { is a } Q \text {-graph } \\
& \Longleftrightarrow s \stackrel{h(v w)}{\rightleftharpoons} t \quad h \text { is a substitution. }
\end{aligned}
$$

ii) Let us prove (b). By restricting (a) to vertices of $h^{-1}(G)$, we can extend it for $u=\varepsilon$ :

$$
s \underset{h^{-1}(G)}{\stackrel{u}{\Longrightarrow}} t \Longleftrightarrow s \underset{G}{\stackrel{h(u)}{\Longrightarrow}} t \text { for any } u \in P^{*} \text { and } s, t \in V_{h^{-1}(G)}
$$

Taking vertex sets $E$ and $F$ of $h^{-1}(G)$, we have

$$
\begin{aligned}
\mathcal{L}\left(h^{-1}(G), E, F\right) & =\left\{u \in P^{*} \mid E \underset{h^{-1}(G)}{\stackrel{u}{\longrightarrow}} F\right\} \\
& =\left\{u \in P^{*} \mid E \underset{G}{\stackrel{h(u)}{\longrightarrow}} F\right\} \\
& =\left\{u \in P^{*} \mid h(u) \in \mathcal{L}(G, E, F)\right\} \\
& =h^{-1}(\mathcal{L}(G, E, F))
\end{aligned}
$$

iii) Let us prove (c). Let $a \in O$ and $s, t \in V_{G}$.

Assume that $\varepsilon \notin g(O) \vee s, t \in V_{h^{-1}(G)}$. We have

$$
\begin{aligned}
& s \underset{g^{-1}\left(h^{-1}(G)\right)}{\stackrel{a}{\longrightarrow}} t \Longleftrightarrow s \underset{h^{-1}(G)}{\stackrel{g(a)}{\rightleftharpoons}} t \quad \text { by definition } \\
& \Longleftrightarrow s \stackrel{h(g(a))}{\longrightarrow} t \quad \text { by }(i) \text { if } \varepsilon \notin g(a) \quad \text { or } \quad \text { by (1) if } s, t \in V_{h^{-1}(G)} \\
& \Longleftrightarrow s \stackrel{(g \circ h)(a)}{\underset{G}{\longrightarrow}} t \\
& \Longleftrightarrow s \underset{(g \circ h)^{-1}(G)}{\stackrel{a}{\longrightarrow}} t \text { by definition }
\end{aligned}
$$

Note that the vertex restriction of Lemma 2.1 (c) is necessary. It is sufficient to take $G=\{1 \stackrel{a}{\longrightarrow} 1,2 \stackrel{b}{\longrightarrow} 2\}$ and the partial morphisms $h(a)=a$ and $g(a)=\varepsilon$ in such a way that $h^{-1}(G)=g^{-1}\left(h^{-1}(G)\right)=\{1 \stackrel{a}{\longrightarrow} 1\}$ and $(g \circ h)^{-1}(G)=\{1 \stackrel{a}{\longrightarrow} 1,2 \stackrel{a}{\longrightarrow} 2\}$.

Another basic property is the commutation between the inverse mapping and the restriction to particular sets. A set $C$ is stable in a graph $G$ when any path between vertices in $C$ contains only vertices in $C$ : 


$$
s_{0} \underset{G}{\longrightarrow} s_{1} \ldots s_{n-1} \underset{G}{\longrightarrow} s_{n} \wedge s_{0}, s_{n} \in C \Longrightarrow s_{1}, \ldots, s_{n-1} \in C
$$

The restriction to any stable set commutes with any inverse mapping.

Lemma 2.2 For any stable set $C$ in a P-graph and any mapping $h$ into $2^{P}$, we have $\quad h^{-1}\left(G_{\mid C}\right)=\left(h^{-1}(G)\right)_{\mid C}$

\section{Proof.}

We have $\quad h^{-1}\left(G_{\mid C}\right)=\left(h^{-1}\left(G_{\mid C}\right)\right)_{\mid C} \subseteq\left(h^{-1}(G)\right)_{\mid C}$.

Let us verify the inverse inclusion.

Let $s \underset{\left(h^{-1}(G)\right)_{\mid C}}{\stackrel{a}{\longrightarrow}} t$. So $s \underset{h^{-1}(G)}{\stackrel{a}{\longrightarrow}} t$ with $s, t \in C$.

By definition of $h^{-1}(G)$, there is $u \in h(a)$ such that $s \stackrel{u}{\Rightarrow} t$.

As $s, t \in C$ and $C$ is stable in $G$, we have $s \underset{G_{\mid C}}{\stackrel{u}{\longrightarrow}} t$. Hence $s \underset{h^{-1}\left(G_{\mid C}\right)}{\stackrel{a}{\longrightarrow}} t$.

Another way to express a restriction of an inverse mapping of a graph is to use a marking of the graph. The marking $\#_{C}(G)$ on a vertex set $C$ of a graph $G$ by a symbol \# is the graph:

$$
\#_{\mathrm{C}}(G):=G \cup\{s \stackrel{\#}{\longrightarrow} s \mid s \in C\}
$$

obtained from $G$ by adding \# to any vertex in $C$. Let us give an example.

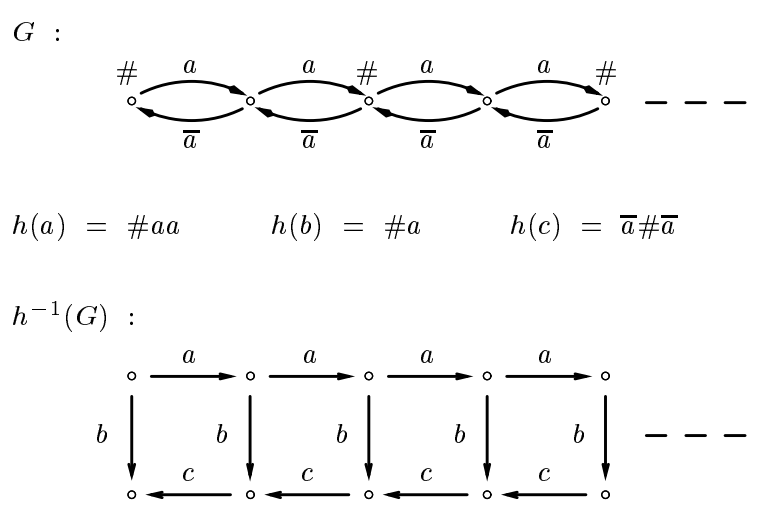

Figure 2.3 An inverse mapping of a marked graph.

Any restriction of an inverse of a graph is an inverse of a marking of the graph.

Lemma 2.4 We have $\left(h^{-1}(G)\right)_{\mid C}=g^{-1}\left(\#_{C}(G)\right)$ with $g(a)=\# h(a) \#$.

\section{Proof.}

Assuming \# is a new symbol and by definition of $g^{-1}$, we have 


$$
\begin{aligned}
g^{-1}\left(\#_{\mathrm{C}}(G)\right) & =\left\{s \stackrel{a}{\longrightarrow} t \mid \exists u \in h(a), s \underset{\#_{\mathrm{C}}(G)}{\stackrel{\# u \#}{\longrightarrow}} t\right\} \\
& =\{s \stackrel{a}{\longrightarrow} t \mid \exists u \in h(a), s \underset{G}{\stackrel{u}{\longrightarrow}} t \wedge s, t \in C\} \\
& =\left(h^{-1}(G)\right)_{\mid C}
\end{aligned}
$$

For $\varepsilon$ the neutral element of the free monoid $T^{*}$ generated by an alphabet $T$, the $\varepsilon$-closure $\bar{G}$ of any $T \cup\{\varepsilon\}$-graph $G$ is obtained by removing the $\varepsilon$-transitions and by adding $T$-transitions as follows:

$$
\bar{G}:=\left(I d_{T}\right)^{-1}(G)=\{s \stackrel{a}{\longrightarrow} t \mid a \in T \wedge s \underset{G}{\stackrel{a}{\longrightarrow}} t\}
$$

Let us give a simple example.

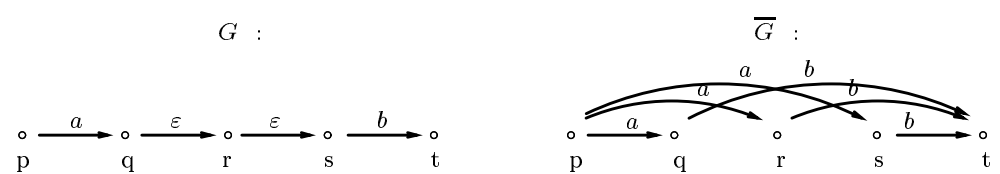

Figure 2.5 E-closure of a graph

We compare graphs by isomorphism. A partial isomorphism from a graph $G$ into

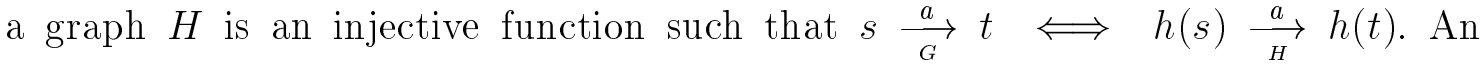
isomorphism is a partial isomorphism such that $V_{G} \subseteq \operatorname{Dom}(h)$ and $V_{H} \subseteq \operatorname{Im}(h)$. A partial isomorphism on $T \cup\{\varepsilon\}$-graphs considers the $\varepsilon$ label as a new letter. To take an $\varepsilon$-transition as an internal (silent) move, we compare $T \cup\{\varepsilon\}$-graphs by partial weak isomorphism. A partial weak isomorphism from a graph $G$ into a graph $H$ is an injective function such that $s \underset{G}{\stackrel{a}{\Rightarrow}} t \Longleftrightarrow h(s) \underset{H}{\stackrel{a}{\Longrightarrow}} h(t)$. A weak isomorphism is a partial weak isomorphism such that $V_{G} \subseteq \operatorname{Dom}(h)$ and $V_{H} \subseteq \operatorname{Im}(h)$. Note that $h$ is a (resp. partial) weak isomorphism from $G$ into $H$ if and only if $h$ is a (resp. partial) isomorphism from $\bar{G}$ into $\bar{H}$. In particular, the identity $I d_{V_{G}}$ is a weak isomorphism between a graph $G$ and $\bar{G}$. Let us give a simple example.
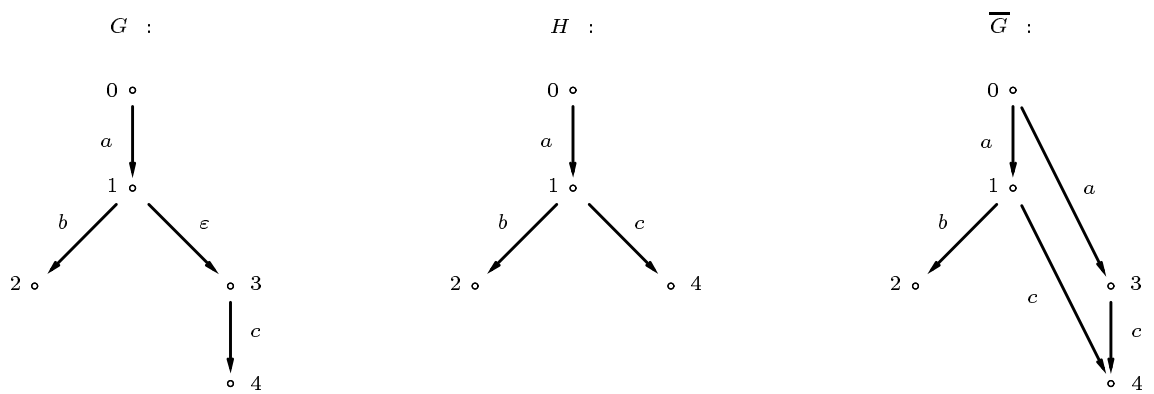

Figure 2.6 $I d_{v_{H}}$ is a partial weak isomorphism between $G$ and $H$ which are not (total) weak isomorphic. 


\section{Classes of graphs}

A general way to define a family $R E C_{F}$ of graphs from a family $F$ of languages is to take the set of graphs obtained from the binary tree (with inverse transitions) by applying an inverse $F$-mapping followed by a rational restriction [Ca 96]. An equivalent way is to take the set of graphs obtained from the binary tree by marking a rational vertex set followed by an inverse $F$-mapping (Proposition 3.5 ). We deduce known results on the family $R E C_{F i n}$ (Theorem 3.6), on the family $R E C_{R a t}$ (Theorem 3.7), on the family $R E C_{\bar{L} \text { in }}$ where $\bar{L}$ in is a subfamily of linear languages (Theorem 3.10). We deduce also closure properties by inverse mappings.

Let $N$ be an alphabet containing at least two letters. We take a new alphabet $\bar{N}:=\{\bar{a} \mid a \in N\}$ in bijection with $N$. We define the following Dyck graph:

$$
\Lambda_{N}:=\left\{u \stackrel{a}{\longrightarrow} u a \mid u \in N^{*} \wedge a \in N\right\} \cup\left\{u a \stackrel{\bar{a}}{\longrightarrow} u \mid u \in N^{*} \wedge a \in N\right\}
$$

where a representation for $N=\{a, b\}$ is the following:

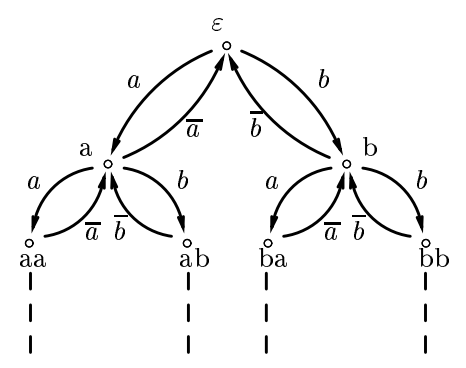

When $L$ is rational, we say that $\#_{L}\left(\Lambda_{N}\right)$ is a rational marking of $\Lambda_{N}$. Note that

$$
x \underset{\#_{w^{-1} L^{\left(\Lambda_{N}\right)}}}{\stackrel{u}{\Longrightarrow}} y \quad \Longrightarrow \quad w x \underset{\#_{L^{\left(\Lambda_{N}\right)}}}{\stackrel{u}{\longrightarrow}} w y
$$

We denote $\mathcal{L}\left(\#_{L}\left(\Lambda_{N}\right)\right):=\mathcal{L}\left(\#_{L}\left(\Lambda_{N}\right), \varepsilon, N^{*}\right)$ the language of path labels of $\Lambda_{N}$ from $\varepsilon$ to all the vertices. When $L$ is rational, $\mathcal{L}\left(\#_{L}\left(\Lambda_{N}\right)\right)$ is a context-free language. Precisely and from a deterministic and complete minimal automaton $(G, \iota, F)$ recognizing $L$, the language $\mathcal{L}\left(\#_{L}\left(\Lambda_{N}\right)\right)$ is generated from axiom $\iota$ by the following context-free grammar:

$\{(p, a q \bar{a} p) \mid p \underset{G}{\stackrel{a}{\rightarrow}} q\} \cup\{(p, a q) \mid p \underset{G}{\stackrel{a}{\rightarrow}} q\} \cup\left\{(p, \varepsilon) \mid p \in V_{G}\right\} \cup\{(p, \# p) \mid p \in F\}$

The binary relation

$$
J:=\{(a \bar{a}, \varepsilon) \mid a \in N\} \cup\{(\#, \varepsilon)\}
$$

has a canonical rewriting, and we write $u \downarrow J$ the irreducible word (normal form) 
that derives from $u$ according to $J$. We have for any word $u \in \mathcal{L}\left(\#_{L}\left(\Lambda_{N}\right)\right)$,

$$
u \downarrow J \in N^{*} \wedge \varepsilon \underset{\#_{L^{\left(\Lambda_{N}\right)}}}{\stackrel{u}{\longrightarrow}} u \downarrow J
$$

The reduction to the set of normal forms preserves the rationality.

Lemma 3.1 Let $L \in \operatorname{Rat}\left(N^{*}\right)$ and $M \in \operatorname{Rat}\left((N \cup \bar{N} \cup\{\#\})^{*}\right)$. We have in an effective way $\left(\mathcal{L}\left(\#_{L}\left(\Lambda_{N}\right)\right) \cap M\right) \downarrow J \in \operatorname{Rat}\left(N^{*}\right)$.

\section{Proof.}

Let $\bar{M}=\left(\mathcal{L}\left(\#_{L}\left(\Lambda_{N}\right)\right) \cap M\right) \downarrow J$.

Let $(G, i, F)$ be a finite $(N \cup \bar{N} \cup\{\#\})$-automaton recognizing $M$.

We color any vertex $u \in N^{*}$ of $\Lambda_{N}$ by the set $c(u)$ of states $p$ such that $(p, u)$ is a vertex of the product $G \times \#_{L}\left(\Lambda_{N}\right)$ accessible from $(i, \varepsilon)$ :

$$
c(u):=\left\{p \mid \mathcal{L}(G, i, p) \cap \mathcal{L}\left(\#_{L}\left(\Lambda_{N}\right), \varepsilon, u\right) \neq \emptyset\right\}
$$

So $\bar{M}=\left\{u \in N^{*} \mid c(u) \cap F \neq \emptyset\right\}$.

We have to show that $\bar{M}$ is rational by proving that $c$ is a regular coloring of $\#_{L}\left(\Lambda_{N}\right)$. We consider the following equivalence $\equiv$ on $N^{*}$ :

$$
u \equiv v \quad \text { if } \quad c(u)=c(v) \wedge u^{-1} L=v^{-1} L
$$

As $\operatorname{Im}(c)$ is finite and $L$ is rational, the equivalence $\equiv$ is of finite index.

This equivalence is right regular: let $u \equiv v$ and $a \in N$. We have to show that $u a \equiv v a$.

As usual $(u a)^{-1} L=a^{-1}\left(u^{-1} L\right)=a^{-1}\left(v^{-1} L\right)=(v a)^{-1} L$.

By symetry of $u$ and $v$, it remains to verify that $c(u a) \subseteq c(v a)$.

Let $p \in c(u a)$. There is $w \in(N \cup \bar{N} \cup\{\#\})^{*}$ such that

$$
i \stackrel{w}{\underset{G}{\Longrightarrow}} p \wedge \varepsilon \underset{\#_{L^{\left(\Lambda_{N}\right)}}}{\stackrel{w}{\Longrightarrow}} u a
$$

Let $x y=w$ such that $\varepsilon \underset{\#_{L^{\left(\Lambda_{N}\right)}}}{\stackrel{x}{\rightleftharpoons}} u \underset{\#_{\left.L^{\left(\Lambda_{N}\right.}\right)}}{\stackrel{y}{\Longrightarrow}} u a$ and $|x|$ is maximal.

By maximality of $|x|$, we have $\varepsilon \underset{\#_{u}-1 L^{\left(\Lambda_{N}\right)}}{\stackrel{y}{\Longrightarrow}} a$.

As $u^{-1} L=v^{-1} L$, we have $\varepsilon \underset{\#_{v^{-1} L^{\left(\Lambda_{N}\right)}}}{\stackrel{y}{\longrightarrow}} a$ hence $v \underset{\#_{L^{\left(\Lambda_{N}\right)}}}{\stackrel{y}{\longrightarrow}} v a$.

There is $q$ such that $i \underset{G}{\stackrel{x}{\Rightarrow}} q \underset{G}{\stackrel{y}{\Rightarrow}} p$. So $q \in c(u)=c(v)$.

By definition of $c(v)$, there is $z \in(N \cup \bar{N} \cup\{\#\})^{*}$ such that

$$
i \underset{G}{\stackrel{z}{\Rightarrow}} q \wedge \varepsilon \underset{\#_{L^{\left(\Lambda_{N}\right)}}}{\stackrel{z}{\Rightarrow}} v
$$

So $i \underset{G}{\stackrel{z y}{\Rightarrow}} p \wedge \varepsilon \underset{\#_{L}\left(\Lambda_{N}\right)}{\stackrel{z y}{\Rightarrow}} v a$. Hence $p \in c(v a)$.

So $H=\left\{[u] \stackrel{a}{\longrightarrow}[u a] \mid u \in N^{*} \wedge a \in N\right\}$ is finite.

Furthermore $\bar{M}=\mathcal{L}(H,[\varepsilon],\{[u] \mid c(u) \cap F \neq \emptyset\})$ is rational.

Let us see that $H$ can be constructed from $(G, i, F)$ and a finite $N$-automaton 
$(\bar{G}, \bar{\iota}, \bar{F})$ recognizing $L$. We may assume that $(\bar{G}, \bar{\iota}, \bar{F})$ is deterministic, complete and minimal. This automaton is isomorphic to the complete (left) residual automaton of $L$ :

$$
\left(\left\{u^{-1} L \stackrel{a}{\longrightarrow}(u a)^{-1} L \mid u \in N^{*} \wedge a \in N\right\}, L,\left\{u^{-1} L \mid \varepsilon \in u^{-1} L\right\}\right)
$$

We denote by $\bar{\imath} \cdot u$ the unique state accessible from $\bar{\imath}$ by the path labelled by $u$ i.e.

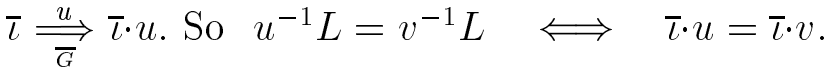

It remains to show that $c(u)$ can be constructed for any $u \in N^{*}$.

Note that $\mathcal{L}\left(\#_{\emptyset}\left(\Lambda_{N}\right), \varepsilon, \varepsilon\right)$ is the context-free language $D_{N}^{\prime}{ }^{*}$. More generally, we will verify that $\mathcal{L}\left(\#_{L}\left(\Lambda_{N}\right), \varepsilon, u\right)$ is an effective context-free language, hence $c(u)$ is computable because the intersection of a rational language with a context-free language is (in an effective way) a context-free language, and the emptyness of a context-free language is decidable (see for instance [Ber 79]).

We define the following context-free grammar:

$$
K:=\{(p, a q \bar{a} p) \mid p \underset{\bar{G}}{\stackrel{a}{\longrightarrow}} q\} \cup\left\{(p, \varepsilon) \mid p \in V_{\bar{G}}\right\} \cup\{(p, \# p) \mid p \in \bar{F}\}
$$

Then $\mathcal{L}\left(\#_{L}\left(\Lambda_{N}\right), \varepsilon, u\right)=\mathcal{L}(K, \bar{\iota} u(1)[\bar{\iota} \cdot u(1)] \ldots u(|u|)[\bar{\iota} \cdot u])$.

Lemma 3.1 with $L=\emptyset$ means that any rational language $M \in \operatorname{Rat}\left((N \cup \bar{N})^{*}\right)$ to mark $\Lambda_{N}$, can be transformed into the following rational language over $N$ :

$$
\left(\mathcal{L}\left(\#_{\emptyset}\left(\Lambda_{N}\right)\right) \cap M\right) \downarrow J=M \downarrow I \cap N^{*}
$$

where $I=\{(a \bar{a}, \varepsilon) \mid a \in N\}$. It is a 'half form' of the standard Benois' lemma [Ben 69].

We denote $\widetilde{u}$ the mirror of any word $u: \widetilde{\varepsilon}=\varepsilon$ and $\widetilde{a u}=\widetilde{u} a$ for any letter $a$. The mapping - associating to any $a \in N$ its barred letter $\bar{a} \in \bar{N}$ is extended by morphism to any word in $(N \cup \bar{N} \cup\{\#\})^{*}$ by defining $\overline{\#}=\#$ and $\overline{\bar{a}}=a$ for any $a \in N$. In this way, we have

$$
s \underset{\#_{L^{\left(\Lambda_{N}\right)}}}{\stackrel{u}{\rightleftharpoons}} t \Longleftrightarrow t \underset{\#_{L^{\left(\Lambda_{N}\right)}}}{\stackrel{\widetilde{\widetilde{u}}}{\longrightarrow}} s
$$

Note also that $\widetilde{\bar{u}}=\overline{\widetilde{u}}$ and $(\widetilde{\bar{u}}) \downarrow J=\widetilde{\overline{u \downarrow J}}$ for any $u \in(N \cup \bar{N} \cup\{\#\})^{*}$.

Any inverse mapping of any marked $\Lambda_{N}$ can be expressed in a suffix way.

Proposition 3.2 For any mapping $h \subseteq T \times(N \cup \bar{N} \cup\{\#\})^{*}$ and any language $L \subseteq N^{*}$, the $T$-graph $h^{-1}\left(\#_{L}\left(\Lambda_{N}\right)\right)$ is equal to

$$
\left\{w(\widetilde{\overline{u \downarrow J}}) \stackrel{a}{\longrightarrow} w(v \downarrow J) \mid u v \in h(a) \wedge \widetilde{\bar{u}}, v \in \mathcal{L}\left(\#_{w^{-1} L}\left(\Lambda_{N}\right)\right) \wedge w \in N^{*}\right\}
$$

\section{Proof.}

Let $G$ be $\left\{w(\widetilde{u \downarrow J}) \stackrel{a}{\longrightarrow} w(v \downarrow J) \mid u v \in h(a) \wedge \widetilde{\bar{u}}, v \in \mathcal{L}\left(\#_{w^{-1}{ }_{L}}\left(\Lambda_{N}\right)\right) \wedge w \in N^{*}\right\}$. We have to show that $h^{-1}\left(\#_{L}\left(\Lambda_{N}\right)\right)=G$. 
i) Proof of $h^{-1}\left(\#_{L}\left(\Lambda_{N}\right)\right) \subseteq G$. Let $s \underset{h^{-1}\left(\#_{\left.L^{(} \Lambda_{N}\right)}\right.}{\stackrel{a}{\longrightarrow}} t$.

There is $z \in h(a)$ such that $s \underset{\#_{L}\left(\Lambda_{N}\right)}{\stackrel{z}{\Rightarrow}} t$.

Let $w \in N^{*}$ of minimal length such that $s \underset{\#_{L^{\left(\Lambda_{N}\right)}}}{\stackrel{u}{\Longrightarrow}} w \underset{\#_{L^{\left(\Lambda_{N}\right)}}}{\stackrel{v}{\longrightarrow}} t$ with $u v=z$ meaning

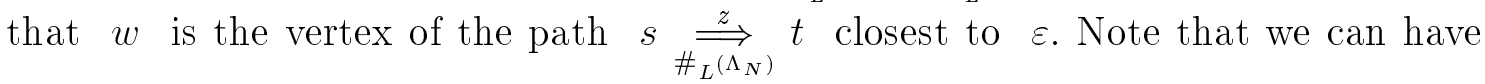
several choices of $(u, v)$. We have $u v \in h(a)$.

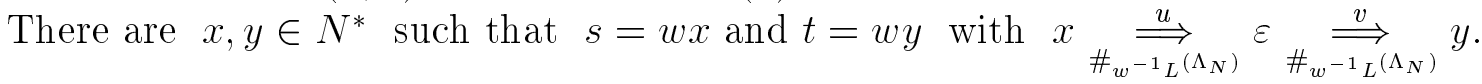

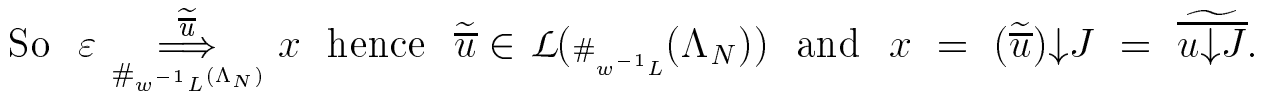

Similarly $v \in \mathcal{L}\left(\#_{w^{-1} L}\left(\Lambda_{N}\right)\right)$ and $y=v \downarrow J$.

Thus $s=w x=w \widetilde{u \downarrow J}$ and $t=w y=w(v \downarrow J)$. Finally $s \underset{G}{\stackrel{a}{\rightarrow}} t$.

ii) Proof of $G \subseteq h^{-1}\left(\#_{L}\left(\Lambda_{N}\right)\right)$. Let $s \underset{G}{\stackrel{a}{\rightarrow}} t$. There are $u v \in h(a)$ and $w \in N^{*}$ such that $\tilde{\bar{u}}, v \in \mathcal{L}\left(\#_{w^{-1} L}\left(\Lambda_{N}\right)\right), s=w(\widetilde{\overline{u \downarrow J}}), t=w(v \downarrow J)$.

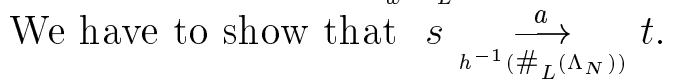

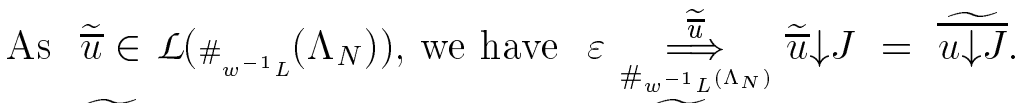

So $\widetilde{u \downarrow J} \underset{\#_{\left.w^{-1} L^{(} \Lambda_{N}\right)}}{\stackrel{u}{\longrightarrow}} \varepsilon$ hence $s=w(\widetilde{u \downarrow J}) \underset{\#_{\left.L^{(} \Lambda_{N}\right)}}{\stackrel{u}{\longrightarrow}} w$.

As $v \in \mathcal{L}\left(\#_{w^{-1} L}\left(\Lambda_{N}\right)\right)$, we have $\varepsilon \underset{\#_{\left.w^{-1} L^{(} \Lambda_{N}\right)}}{\stackrel{v}{\longrightarrow}} v \downarrow J$ hence $\underset{\#_{L^{\left(\Lambda_{N}\right)}}}{\stackrel{v}{\longrightarrow}} w(v \downarrow J)=t$.

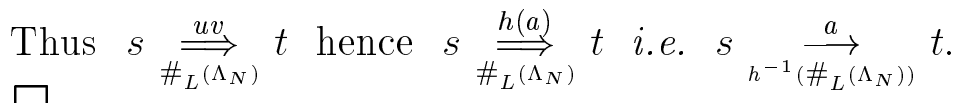

To give a simple form of Proposition 3.2 for any rational marking, we introduce some notations. The right concatenation of a graph $G \subseteq N^{*} \times T \times N^{*}$ by a language $L \subseteq N^{*}$ is the following graph:

$$
G . L:=\{u w \stackrel{a}{\longrightarrow} v w \mid u \stackrel{a}{\rightarrow} v \wedge w \in L\}
$$

Similarly, we define the left concatenation $L . G$ of a graph $G$ by a language $L$. A usual and simple fact is that for any $L \in \operatorname{Rat}\left(N^{*}\right)$ and any $u \in N^{*}$, the language $[u]_{L}:=\left\{v \mid v^{-1} L=u^{-1} L\right\} \in \operatorname{Rat}\left(N^{*}\right)$ and the family $[L]:=\left\{[u]_{L} \mid u \in N^{*}\right\}$ is finite. Note that for any $W \in[L]$ and any $w \in W, W^{-1} L=w^{-1} L$.

From Proposition 3.2, it follows that any inverse mapping of any rational marked $\Lambda_{N}$ is a finite union of graphs $W . H$ with $W \in \operatorname{Rat}\left(N^{*}\right)$.

Corollary 3.3 For any $h \subseteq T \times(N \cup \bar{N} \cup\{\#\})^{*}$ and $L \in \operatorname{Rat}\left(N^{*}\right)$, $h^{-1}\left(\#_{L}\left(\Lambda_{N}\right)\right)=\bigcup_{W \in[L]} W \cdot\left\{\widetilde{u \downarrow J} \stackrel{a}{\longrightarrow} v \downarrow J \mid u v \in h(a) \wedge \widetilde{u}, v \in \mathcal{L}\left(\#_{W^{-1} L}\left(\Lambda_{N}\right)\right)\right\}$ 
To get a prefix form of Corollary 3.3, we take the mirror $\widetilde{\Lambda}_{N}$ of $\Lambda_{N}$ where

$$
\widetilde{G}=\{\widetilde{u} \stackrel{a}{\longrightarrow} \widetilde{v} \mid u \underset{G}{\stackrel{a}{\longrightarrow}} v\} \text { is the mirror of graph } G \subseteq N^{*} \times T \times N^{*}
$$

By applying Corollary 3.3 to $\#_{L}\left(\widetilde{\Lambda}_{N}\right)=\left(\widetilde{\#_{\widetilde{L}}\left(\Lambda_{N}\right)}\right)$, we have for every mapping $h \subseteq T \times(N \cup \bar{N} \cup\{\#\})^{*}$ and for every $L \in \operatorname{Rat}\left(N^{*}\right)$,

$$
h^{-1}\left(\#_{L}\left(\widetilde{\Lambda}_{N}\right)\right)=\bigcup_{W \in[L]}\left\{\overline{u \downarrow J} \stackrel{a}{\rightarrow} \widetilde{v \downarrow J} \mid u v \in h(a) \wedge \widetilde{\bar{u}}, v \in L\left(\#_{W^{-1}} \widetilde{L}\left(\Lambda_{N}\right)\right)\right\} . \widetilde{W}
$$

Proposition 3.2 has also a simple form when we do not use marking.

Corollary 3.4 For any mapping $h \subseteq T \times(N \cup \bar{N})^{*}$, we have the T-graph $h^{-1}\left(\Lambda_{N}\right)=N^{*} \cdot\left\{u \stackrel{a}{\longrightarrow} v \mid \widetilde{\bar{u}} v \in h(a) \downarrow I \wedge u, v \in N^{*} \wedge a \in T\right\}$

Let $D y c k_{N}=\left[\Lambda_{N}\right]$ where $[G]$ is the set of all graphs isomorphic to a graph $G$, that we extend by union to any class of graphs. We restrict here a language family $F$ to be a subset of $2^{(N \cup \bar{N} \cup\{\#\})^{*}}$. A family of languages defines a set of mappings: a mapping $h$ is rational (resp linear, ...) if for any letter $a$, the language $h(a)$ is rational (resp linear, ...). Precisely, a language family $F$ and an alphabet $T$ produce the set $F_{T}$ of mappings defined for every $a \in T$ by a language $h(a) \in F$. By inverse of a class $\Phi$ of $(N \cup \bar{N} \cup\{\#\})$-graphs, we get the following class of $T$-graphs:

$$
F_{T}^{-1}(\Phi):=\left\{h^{-1}(G) \mid G \in \Phi \wedge h \in F_{T}\right\}
$$

Starting from $D y c k_{N}$, we have two ways to get classes of graphs. Either we apply inverse $F$-mappings followed by rational restrictions [Ca 96] :

$$
F_{T}^{-1}\left(\operatorname{Dyck}_{N}\right) \mid:=\left[\left\{h^{-1}\left(\Lambda_{N}\right)_{\mid L} \mid h \in F_{T} \wedge L \in \operatorname{Rat}\left(N^{*}\right)\right\}\right]
$$

or we apply rational markings followed by inverse $F$-mappings:

$$
F_{T}^{-1}\left(\#\left(\text { Dyck }_{N}\right)\right):=\left[\left\{h^{-1}\left(\#_{L}\left(\Lambda_{N}\right)\right) \mid h \in F_{T} \wedge L \in \operatorname{Rat}\left(N^{*}\right)\right\}\right]
$$

Henceforth $F$ will be one of the following language families: the family Fin of finite languages; the family Rat of rational languages; the family Lin of linear languages; the family $R E$ of recursively enumerable languages; the subfamily $\bar{L}$ in of linear languages generated by linear grammars such that each right hand side is $\varepsilon$ or of the form $u B v$ where $B$ is a non-terminal with $u \in \bar{N}^{*}$ and $v \in N^{*}$; and the rational closure $\bar{L}$ in $(R a t)$ of $\bar{L}$ in. Each of these families is an internal family meaning that it satisfies the two following conditions: 
(ii) $L \in F \Longrightarrow h(L) \downarrow J \cap \bar{N}^{*} N^{*} \in F$

for any finite substitution $h$ from $(N \cup \bar{N} \cup\{\#\})^{*}$ into itself such that $h(\#) \downarrow \widetilde{J} \subseteq\{\varepsilon\}$ and for any $a \in N, h(\bar{a})=\widetilde{\overline{h(a)}}$ and $h(a) \downarrow \widetilde{J} \subseteq N^{*}$.

Note that any family closed by every rational binary relation and called a rational cone [Ber 79], is an internal family. For these families, the two previous classes of graphs coincide and we can also restrict $N$ to have only two letters.

Proposition 3.5 For any distinct letters $a, b \in N$ and for any internal family $F$, we have

$F_{T}^{-1}\left(\text { Dyck }_{N}\right)_{\mid}=F_{T}^{-1}\left(\text { Dyck }_{\{a, b\}}\right)_{\mid}=F_{T}^{-1}\left(\#\left(\right.\right.$ Dyck $\left.\left._{\{a, b\}}\right)\right)=F_{T}^{-1}\left(\#\left(\right.\right.$ Dyck $\left.\left._{N}\right)\right)$

This class is denoted $R E C_{F_{T}}$ or $R E C_{F}$ when $T$ is undertood.

\section{Proof.}

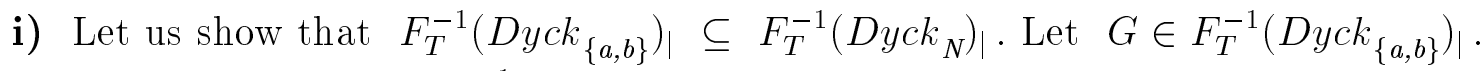
So $G$ is isomorphic to $h^{-1}\left(\Lambda_{\{a, b\}}\right)_{\mid L}$ with $h \in F_{T}$ and $L \in \operatorname{Rat}\left(N^{*}\right)$.

We have $\Lambda_{\{a, b\}}=\iota^{-1}\left(\Lambda_{N}\right)_{\mid\{a, b\}^{*}}$ where $\iota=I d_{\{a, b, \bar{a}, \bar{b}\}}$.

Note that $\{a, b\}^{*}$ is stable for $\iota^{-1}\left(\Lambda_{N}\right)$. Henceforth $G$ is isomorphic to

$$
\begin{array}{rlrl}
h^{-1}\left(\iota^{-1}\left(\Lambda_{N}\right)_{\mid\{a, b\}^{*}}\right)_{\mid L} & =\left(h^{-1}\left(\iota^{-1}\left(\Lambda_{N}\right)\right)_{\mid\{a, b\}^{*}}\right)_{\mid L} & & \text { by Lemma } 2.2 \\
& =(h \circ \iota)^{-1}\left(\Lambda_{N}\right)_{\mid V_{\iota^{-1}\left(\Lambda_{N}\right)} \cap\{a, b\}^{*} \cap L} & & \text { by Lemma } 2.1(\mathrm{c}) \\
& =(h \circ \iota)^{-1}\left(\Lambda_{N}\right)_{\mid\{a, b\}^{*} \cap L} &
\end{array}
$$

By condition (ii) of an internal family $F$ (restricted to partial morphism), we have $h \circ \iota \in F_{T}$ hence $G \in F_{T}^{-1}\left(D_{y c k}\right)$ |

ii) Let us show that $F_{T}^{-1}\left(D y c k_{N}\right) \mid \subseteq F_{T}^{-1}\left(\#\left(D y c k_{N}\right)\right)$. Let $G \in F_{T}^{-1}\left(D y c k_{N}\right)$. So $G$ is isomorphic to $h^{-1}\left(\Lambda_{N}\right)_{\mid L}$ with $h \in F_{T}$ and $L \in \operatorname{Rat}\left(N^{*}\right)$.

By Lemma 2.4, $h^{-1}\left(\Lambda_{N}\right)_{\mid L}=g^{-1}\left(\#_{L}\left(\Lambda_{N}\right)\right)$ with $g(a)=\# h(a) \#$ for any $a \in T$. By condition $(i)$ of an internal family $F$, we have $g \in F_{T}$ hence $G \in F_{T}^{-1}\left(\#\left(D y c k_{N}\right)\right)$.

iii) Let us show that $F_{T}^{-1}\left(\#\left(D y c k_{N}\right)\right) \subseteq F_{T}^{-1}\left(D y c k_{\{a, b\}}\right)$. Let $G \in F_{T}^{-1}\left(\#\left(D y c k_{N}\right)\right)$. So $G$ is isomorphic to $h^{-1}\left(\#_{L}\left(\Lambda_{N}\right)\right)$ with $h \in F_{T}$ and $L \in \operatorname{Rat}\left(N^{*}\right)$.

Let $(A, i, F)$ be a finite deterministic and complete automaton recognizing $L$ and such that $p \underset{A}{\stackrel{c}{\longrightarrow}} q \wedge p \underset{A}{\stackrel{d}{\longrightarrow}} q \Longrightarrow c=d$. By duplication of states, it is easy to satisfy this condition. However this condition is not necessary but it permits to simplify the notations.

Let $Q=V_{A}$ be the state set of $A$ and let $P$ be the language of words obtained from $i$ by suffix derivation according to the relation $\{(p, p q) \mid \exists a \underset{A}{p} \underset{a}{\rightarrow} q\}$. From [Bü 64], $P \in \operatorname{Rat}\left(N^{*}\right)$. 
So $\#_{L}\left(\Lambda_{N}\right)$ is isomorphic to $f^{-1}\left(\Lambda_{Q}\right)_{\mid P}$ where $f$ is the following finite mapping:

$$
f(a)=\{\bar{p} p q \mid p \underset{A}{\stackrel{a}{\rightarrow}} q\} ; f(\bar{a})=\{\bar{q} \bar{p} p \mid p \underset{A}{\stackrel{a}{\rightarrow}} q\} ; f(\#)=\{\bar{p} p \mid p \in F\}
$$

By definition, $P$ is the vertex set of the connected component of $f^{-1}\left(\Lambda_{Q}\right)$ containing $i$, hence $P$ is stable for $f^{-1}\left(\Lambda_{Q}\right)$. Note that $Q$ may have more than two letters. As for (i), we denote $Q=\left\{a_{1}, \ldots, a_{n}\right\}$ and we have

$$
g\left\lfloor\Lambda_{Q}\right\rfloor=g^{-1}\left(\Lambda_{\{a, b\}}\right)_{\mid M} \text { with } \quad \begin{aligned}
& g\left(a_{i}\right)=a b^{i-1} \text { and } g\left(\bar{a}_{i}\right)=\bar{b}^{i-1} \bar{a} \text { for } i \in[n] \\
& M=g\left(\left\{a_{1}, \ldots, a_{n}\right\}^{*}\right)=\left\{a, \ldots, a b^{n-1}\right\}^{*}
\end{aligned}
$$

Note that $M$ is stable for $g^{-1}\left(\Lambda_{\{a, b\}}\right)$. Henceforth $\#_{L}\left(\Lambda_{N}\right)$ is isomorphic to

$$
\begin{aligned}
& g\left\lfloor f^{-1}\left(\Lambda_{Q}\right)_{\mid P}\right\rfloor=g\left\lfloor f^{-1}\left(\Lambda_{Q}\right)\right\rfloor_{\mid g(P)} \\
& =f^{-1}\left(g\left\lfloor\Lambda_{Q}\right\rfloor\right) \mid g(P) \\
& =f^{-1}\left(g^{-1}\left(\Lambda_{\{a, b\}}\right)_{\mid M}\right)_{\mid g(P)} \\
& =f^{-1}\left(g^{-1}\left(\Lambda_{\{a, b\}}\right)\right)_{\mid M \cap g(P)} \quad \text { by Lemma } 2.2 \\
& =(f \circ g)^{-1}\left(\Lambda_{\{a, b\}}\right)_{\mid V_{g^{-1}\left(\Lambda_{\{a, b\}}\right)} \cap M \cap g(P)} \quad \text { by Lemma } 2.1 \text { (c) } \\
& =(f \circ g)^{-1}\left(\Lambda_{\{a, b\}}\right)_{\mid g(P)}
\end{aligned}
$$

Note that $g(P)$ is stable for $(f \circ g)^{-1}\left(\Lambda_{\{a, b\}}\right)$. By Lemma 2.2, $G$ is isomorphic to $h^{-1}\left((f \circ g)^{-1}\left(\Lambda_{\{a, b\}}\right)_{\mid g(P)}\right)=(h \circ f \circ g)^{-1}\left(\Lambda_{\{a, b\}}\right)_{\mid g(P)}=((h \circ f \circ g) \downarrow J)^{-1}\left(\Lambda_{\{a, b\}}\right)_{\mid g(P)}$ where for any $x \in T,(h \circ f \circ g) \downarrow J(x)=((f \circ g)(h(x))) \downarrow J$.

As $f \circ g:(N \cup \bar{N} \cup\{\#\})^{*} \longrightarrow 2^{\{a, b, \bar{a}, \bar{b}\}^{*}}$ is a finite substitution, and by condition (ii) of an internal family $F$, we have $(h \circ f \circ g) \downarrow J \in F_{T}$ hence $G$ belongs to $F_{T}^{-1}\left(D_{\left.y c k_{\{a, b\}}\right) \mid}\right.$.

iv) By (i), (ii), (iii), we have

$F_{T}^{-1}\left(\text { Dyck }_{\{a, b\}}\right)_{\mid} \subseteq F_{T}^{-1}\left(\right.$ Dyck $\left._{N}\right) \mid \subseteq F_{T}^{-1}\left(\#\left(\right.\right.$ Dyck $\left.\left._{N}\right)\right) \subseteq F_{T}^{-1}\left(\right.$ Dyck $\left._{\{a, b\}}\right) \mid$

Hence $F_{T}^{-1}\left(D y c k_{\{a, b\}}\right)_{\mid}=F_{T}^{-1}\left(D y c k_{N}\right)_{\mid}=F_{T}^{-1}\left(\#\left(D y c k_{N}\right)\right)$.

For $N=\{a, b\}$, the last equation is $F_{T}^{-1}\left(D y c k_{\{a, b\}}\right) \mid=F_{T}^{-1}\left(\#\left(D y c k_{\{a, b\}}\right)\right)$.

The class $R E C_{\text {Fin }}$ is the set of regular graphs (see [MuS 85], [Co 90], [Ca 90]) of bounded degree, and we present again two sets of representatives.

Theorem 3.6 [Ca 95] [Ca 96] Given an alphabet $N$ of at least two letters, the following properties are equivalent:

a) $G \in R E C_{\text {Fin }_{T}}$

b) $G$ is isomorphic to $\left(H . N^{*}\right)_{\mid L}$ for some finite $H \subseteq N^{*} \times T \times N^{*}$ and 
$L \in \operatorname{Rat}\left(N^{*}\right)$

c) $G$ is isomorphic to $\bigcup_{i=1}^{n}\left(u_{i} \stackrel{a_{i}}{\longrightarrow} v_{i}\right) . W_{i}$ for some $n \geq 0, a_{1}, \ldots, a_{n} \in T$, $u_{1}, v_{1}, \ldots, u_{n}, v_{n} \in N^{*}, W_{1}, \ldots, W_{n} \in \operatorname{Rat}\left(N^{*}\right)$

d) $G$ is a regular $T$-graph of bounded degree.

The traces of the graphs in $R E C_{F i n}$ are all the context-free languages.

\section{Proof.}

i) $(a) \Longrightarrow(b)$ : Let $G \in R E C_{F_{i n}}$.

We have $G$ isomorphic to $h^{-1}\left(\widetilde{\Lambda}_{N}\right)_{\mid L}$ for some $h \in \operatorname{Fin}_{T}$ and $L \in \operatorname{Rat}\left(N^{*}\right)$.

Taking the finite graph $H=\{u \stackrel{a}{\rightarrow} v \mid a \in T \wedge \bar{u} \widetilde{v} \in h(a) \downarrow I\}$ and by Corollary 3.4, we have $h^{-1}\left(\widetilde{\Lambda}_{N}\right)=H . N^{*}$.

ii) $(b) \Longrightarrow(a)$ : Let a finite graph $H \subseteq N^{*} \times T \times N^{*}$ and $L \in \operatorname{Rat}\left(N^{*}\right)$.

By Corollary 3.4, $H=h^{-1}\left(\widetilde{\Lambda}_{N}\right)$ such that $h(a)=\{\bar{u} \widetilde{v} \mid u \underset{H}{\rightarrow} v\} \forall a \in T$.

iii) $(a) \Longrightarrow(c)$ : Let $G \in R E C_{F_{i n}}$.

So $G$ is isomorphic to $h^{-1}\left(\#_{L}\left(\widetilde{\Lambda}_{N}\right)\right)$ with $h \in F_{i n}$ and $L \in \operatorname{Rat}\left(N^{*}\right)$.

It remains to apply the equation (2) which is the prefix form of Corollary 3.3.

iv) $(c) \Longrightarrow(a)$ : Let $n \geq 0, a_{1}, \ldots, a_{n} \in T, u_{1}, v_{1}, \ldots, u_{n}, v_{n} \in N^{*}, W_{1}, \ldots, W_{n} \in$ $\operatorname{Rat}\left(N^{*}\right)$. Let us show that $G=\bigcup_{i=1}^{n}\left(u_{i} \stackrel{a_{i}}{\longrightarrow} v_{i}\right) . W_{i}$ is in $R E C_{\text {Fin }_{T}}$.

Taking the following rational language $L$ and the following finite mapping $h$ :

$$
L=\bigcup_{i=1}^{n} a_{i} W_{i} \quad \text { and } \quad h(a)=\left\{\bar{u}_{i} a_{i} \# \bar{a}_{i} \widetilde{v}_{i} \mid a_{i}=a\right\} \text { for every } a \in T
$$

we have $G=h^{-1}\left(\#_{L}\left(\widetilde{\Lambda}_{N \cup T}\right)\right)$ in $R E C_{F i n}$.

Recall that a graph $G \subseteq N^{*} \times T \times N^{*}$ is recognizable if $G$ is a finite union of elementary graphs of the form $U \stackrel{a}{\rightarrow} V$ where $a \in T$ and $U, V \in \operatorname{Rat}\left(N^{*}\right)$. The class $R E C_{R a t}$ has been studied in [Ca 96] and we present again two sets of representatives.

Theorem 3.7 [Ca 96] Given an alphabet $N$ of at least two letters, the following properties are equivalent:

a) $G \in R E C_{R a t}$

b) $G$ is isomorphic to $\left(H . N^{*}\right)_{\mid L}$ for some recognizable $H \subseteq N^{*} \times T \times N^{*}$ and $L \in \operatorname{Rat}\left(N^{*}\right)$

c) $G$ is isomorphic to $\bigcup_{i=1}^{n}\left(U_{i} \stackrel{a_{i}}{\longrightarrow} V_{i}\right) . W_{i}$ for some $n \geq 0, a_{1}, \ldots, a_{n} \in T$, $U_{1}, V_{1}, W_{1}, \ldots, U_{n}, V_{n}, W_{n} \in \operatorname{Rat}\left(N^{*}\right)$.

The traces of the graphs in $R E C_{\text {Rat }}$ are all the context-free languages.

\section{Proof.}

The implications $(b) \Longrightarrow(a)$ and $(c) \Longrightarrow(a)$ are as in the proof of Theorem 3.6. 
i) $(a) \Longrightarrow(b)$ : as in the proof of Theorem 3.6, it remains to verify that for any $L \in \operatorname{Rat}\left((N \cup \bar{N})^{*}\right), L \downarrow I \cap \bar{N}^{*} N^{*}$ is a finite union of sets of the form $A$. B where $A \in \operatorname{Rat}\left(\bar{N}^{*}\right)$ and $B \in \operatorname{Rat}\left(N^{*}\right)$.

Let $(A, i, F)$ be a finite automaton recognizing $L$ and let $Q=V_{A}$ be the state set of $A$. We have

$$
L \downarrow I \cap \bar{N}^{*} N^{*}=\bigcup_{q \in Q}\left(\overline{\mathcal{L}} \overline{(G, i, q)} \cap \mathcal{L}\left(\Lambda_{N}\right)\right) \downarrow I .\left(\mathcal{L}(\widetilde{G, q, F}) \cap \mathcal{L}\left(\Lambda_{N}\right)\right) \downarrow I
$$

and the rationality follows from Lemma 3.1 .

ii) $(a) \Longrightarrow(c)$ : Let $G \in R E C_{\text {Rat }_{T}}$.

So $G$ is isomorphic to $h^{-1}\left(\#_{L}\left(\widetilde{\Lambda}_{N}\right)\right)$ with $h \in \operatorname{Rat}_{T}$ and $L \in \operatorname{Rat}\left(N^{*}\right)$.

For every $a \in T$, let $\left(A_{a}, i_{a}, F_{a}\right)$ be a finite automaton recognizing $h(a)$ and let $Q_{a}=V_{A_{a}}$ be the state set of $A_{a}$.

By applying the equation (2) which is the prefix form of Corollary $3.3, h^{-1}\left(\#_{L}\left(\widetilde{\Lambda}_{N}\right)\right)$ is equal to

$\bigcup_{\substack{W \in[L] \\ a \in T \\ q \in Q_{a}}}\left(\left(\overline{\mathcal{L}} \overline{\left(G_{a}, i_{a}, q\right)} \cap \mathcal{L}\left(\#_{W^{-1} \widetilde{L}}\left(\Lambda_{N}\right)\right)\right) \downarrow J \stackrel{a}{\longrightarrow}\left(\mathcal{L}\left(\widetilde{G_{a}, q, F_{a}}\right) \cap \mathcal{L}\left(\#_{W^{-1} \widetilde{L}}\left(\Lambda_{N}\right)\right)\right) \downarrow J\right) . \widetilde{W}$

and the rationality follows from Lemma 3.1 .

Several characterizations of $R E C_{F i n}$ inside $R E C_{R a t}$ have been given [Ba 98], [CaK 01]. A major question is the closure of $R E C_{F}$ by inverse $F$-mappings. We denote by

$$
F(E):=\left\{h(L) \mid L \in E \wedge h \in F_{N \cup \bar{N} \cup\{\#\}}\right\}
$$

the family obtained by applying $F$ substitutions to a family $E$. In particular Fin $(F i n)=$ Fin and $F$ in $($ Rat $)=\operatorname{Rat}(F i n)=\operatorname{Rat}($ Rat $)=$ Rat.

Lemma 3.8 Let $F$ be the internal family Fin or Rat. Let $E$ be any family such that $F(E)$ is internal. We have $E_{T}^{-1}\left(R E C_{F_{N}}\right) \subseteq R E C_{F(E)_{T}}$.

\section{Proof.}

i) Let $G \in E_{T}^{-1}\left(R E C_{F_{N}}\right): G=g^{-1}(H)$ for some $g \in E_{T}$ and $H \in R E C_{F_{N}}$.

So $H$ is isomorphic to $h^{-1}\left(\#_{L}\left(\Lambda_{N}\right)\right)$ with $h \in F_{N}$ and $L \in \operatorname{Rat}\left(N^{*}\right)$.

Hence $G$ is isomorphic to $g^{-1}\left(h^{-1}\left(\#_{L}\left(\Lambda_{N}\right)\right)\right)$.

By Lemma 2.1 (c), $G$ is isomorphic to $(g \circ h)^{-1}\left(\#_{L}\left(\Lambda_{N}\right)\right)_{\mid V_{h^{-1}}\left(\#_{L}\left(\Lambda_{N}\right)\right)}$.

For any $a \in T,(g \circ h)(a)=h(g(a)) \in F(E)$ hence $(g \circ h)^{-1}\left(\#_{L}\left(\Lambda_{N}\right)\right) \in$ $R E C_{F(E)_{T}}$.

So $(g \circ h)^{-1}\left(\#_{L}\left(\Lambda_{N}\right)\right)$ is isomorphic to $k^{-1}\left(\Lambda_{N}\right)_{\mid M}$ with $k \in F(E)_{T}$ and $M \in$ $\operatorname{Rat}\left(N^{*}\right)$.

Finally $G$ is isomorphic to $k^{-1}\left(\Lambda_{N}\right)_{\left.\mid M \cap V_{h^{-1}\left(\#_{L}(\Lambda\right.} N^{)}\right)}$.

ii) It remains to show that $V_{h^{-1}\left(\#_{L}\left(\Lambda_{N}\right)\right)}$ is rational. By union, it is sufficient to 
assume that $h(a) \in$ Rat for some $a \in T$ and $h(b)=\emptyset$ for any $b \in T-\{a\}$.

So $h(a)=\mathcal{L}\left(A, i, Q_{f}\right)$ is recognized by some finite automaton $\left(A, i, Q_{f}\right)$. Let $Q=V_{A}$ be the state set of the automaton. By Corollary 3.3, we have

$$
V_{h^{-1}\left(\#_{L}\left(\Lambda_{N}\right)\right)}=\bigcup_{\substack{W \in[L] \\ q \in C(Q)}} W \cdot\left(\left[\overline{\mathcal{L}} \widetilde{(\overline{G, i, q)}} \cup \mathcal{L}\left(G, q, Q_{f}\right)\right] \cap \mathcal{L}\left(\#_{W^{-1} L^{-}}\left(\Lambda_{N}\right)\right)\right) \downarrow J
$$

where $C(Q)$ is the set $q \in Q$ such that the languages $\overline{\mathcal{L}} \widetilde{(G, i, q)} \cap \mathcal{L}\left(\#_{W^{-1} L_{L}}\left(\Lambda_{N}\right)\right)$ and $\mathcal{L}\left(G, q, Q_{f}\right) \cap \mathcal{L}\left(\#_{W^{-1} L}\left(\Lambda_{N}\right)\right)$ are non empty. The rationality follows from Lemma 3.1 .

We deduce closure properties for $R E C_{F i n}$ and $R E C_{R a t}$.

$$
\begin{aligned}
& \text { Proposition } 3.9 \text { We have } \operatorname{Fin}_{T}^{-1}\left(R E C_{F_{i n}}\right)=R E C_{F_{i n}} \\
& \text { and } \operatorname{Rat}_{T}^{-1}\left(R E C_{\text {Fin }_{N}}\right)=\operatorname{Rat}_{T}^{-1}\left(R E C_{\text {Rat }_{N}}\right)=R E C_{\text {Rat }_{T}}
\end{aligned}
$$

\section{Proof.}

As $\#\left(D y c k_{\{a, b\}}\right) \in R E C_{F_{i n}}$, we have

$$
R E C_{F_{i n}}=\operatorname{Fin}_{T}^{-1}\left(\#\left(D_{y c k_{\{a, b\}}}\right)\right) \subseteq \operatorname{Fin}_{T}^{-1}\left(R E C_{F i n_{N}}\right) .
$$

As $F i n(F i n)=$ Fin and by Lemma 3.8, we have Fin $_{T}^{-1}\left(R E C_{F i n_{N}}\right) \subseteq R E C_{F i n_{T}}$. Similarly, we deduce the two other equalities.

Note that the closure of $R E C_{R a t}$ by any inverse rational mapping has been obtained in [Ca 96] with a long proof.

It remains to recall the family of rational graphs [Mo 00]. We consider a graph as a subset of $N^{*} \times T \times N^{*}$ i.e. a $T$-graph with vertices in $N^{*}$. We extend the monoid $N^{*} \times N^{*}$ to the partial semigroup $N^{*} \times T \times N^{*}$ defined by $(u, a, v) \cdot(x, a, y)=(u x, a, v y)$ for every $u, v, x, y \in N^{*}$ and $a \in T$. The extension by union of . to subsets is the usual synchronization product for graphs [AN 82]:

$$
G . H=\{u x \stackrel{a}{\rightarrow} v y \mid u \stackrel{a}{\rightarrow} v \wedge x \underset{H}{\rightarrow} y\} \quad \text { for any } G, H \subseteq N^{*} \times T \times N^{*}
$$

To this operation is associated the rational family $\operatorname{Rat}\left(N^{*} \times T \times N^{*}\right)$ of graphs: it is the smallest subset of $2^{N^{*} \times T \times N^{*}}$ containing the finite graphs and closed by $\cup, \ldots,+$. A rational graph is a graph isomorphic to a graph in $\operatorname{Rat}\left(N^{*} \times T \times N^{*}\right)$; we denote by $R A T_{T}$ the family of rational $T$-graphs. The rational graphs are the graphs recognized by the labelled transducers. Precisely, a T-labelled transducer is a finite $\left(N^{*} \times N^{*}\right)$ automaton $A=\left(G, i,\left(F_{a}\right)_{a \in T}\right)$ with a set $F_{a}$ of final states for each $a \in T$; such an automaton recognizes the graph: 


$$
\mathcal{L}(A):=\left\{u \stackrel{a}{\longrightarrow} v \mid \exists s \in F_{a}, i \stackrel{u / v}{\longrightarrow} s\right\}
$$

The family $\bar{L}$ in defines by inverse mappings the class of rational graphs.

Theorem 3.10 [Mo 00], [MoS 01] We have

$$
R A T_{T}=R E C_{\overline{L i n}_{T}} \subset R E C_{L_{i n}}
$$

The traces of the graphs in $R A T$ are the context-sensitive languages.

A particular rational graph is an automatic graph [BG 00] which is a graph isomorphic to a graph recognized by a labelled left-synchronized (or by a labelled right-synchronized) transducer [EM 65], [FS 93]. The traces of the automatic graphs remain the context-sensitive languages [Ri 01]. Note that we can have non recursive traces for graphs in $R E C_{\text {Lin }}$. From the closure by composition of rational relations, the rational graphs are closed by inverse finite mappings.

Proposition 3.11 We have $\operatorname{Fin}_{T}^{-1}\left(\right.$ RAT $\left._{N}\right)=R A T_{T}$.

We will now use Turing machines to define a general class of graphs whose the traces are the recursively enumerable languages.

\section{Graphs of rewriting systems and of Turing machines}

We consider the rational restrictions of the $\varepsilon$-closure for the set of transitions of the labelled Turing machines. We show that this family is the same that for the labelled word rewriting systems (Theorem 4.5). We show also that this family is $R E C_{F}$ for any family $F$ of recursively enumerable languages containing the rational closure of the linear languages (Theorem 4.6). Furthermore, we show that this family is the set of the inverse rational mappings of the rational graphs (Theorem 4.7). Finally, we show that this family is also the set of graphs recognized by (unlabelled) Turing machines with labelled final states (Theorem 4.8), and even if we restrict to deterministic Turing machines (Theorem 4.9).

The notion of a word-rewriting system is well-known (see for instance the survey [DJ 90] and [BO 93]): it is just a finite set of rules between words. As for the transitions of a pushdown automaton, we allow labelled rules, and to any system, we associate a rational language of admissible words, usually called configurations, which are the words where the rules can be applied. The words are over an alphabet (finite set of symbols) $N$ of non-terminals, and the rules are labelled by symbols in an 
alphabet $T$ of terminals, plus the empty word $\varepsilon$.

Definition 4.1 A finite labelled rewriting system $(R, C)$ over words is a couple of a finite relation $R \subseteq N^{*} \times(T \cup\{\varepsilon\}) \times N^{*}$ and a rational language $C \subseteq N^{*}$ of configurations. We write shortly $R$ instead of $\left(R, N^{*}\right)$.

The set of transitions of $R$ is the following $(T \cup\{\varepsilon\})$-graph:

$$
T(R):=\left\{x u y \stackrel{a}{\longrightarrow} x v y \mid(u, a, v) \in R \wedge x, y \in N^{*}\right\}
$$

The unlabelled transitions of $T(R)$ form the usual rewriting $\underset{R}{\longrightarrow}$ of $R$ :

$$
x u y \underset{R}{\longrightarrow} \text { xvy for some }(u, a, v) \in R \text { with } x, y \in N^{*}
$$

Its reflexive and transitive closure $\longrightarrow_{R}^{*}$ by composition is the derivation of $R$. To any system $(R, C)$, we associate its transition graph:

$$
G(R, C):=\overline{T(R)}_{\mid C}=\{u \stackrel{a}{\longrightarrow} v \mid u \underset{T(R)}{\stackrel{a}{\longrightarrow}} v \wedge u, v \in C \wedge a \in T\}
$$

which is the restriction to $C$ of the $\varepsilon$-closure of $T(R)$. In particular $G(R)=\overline{T(R)}$. For instance, the transition relation of a pushdown automaton over a set $Q$ of states and over a disjoint set $P$ of stack letters, can be seen as a labelled rewriting system $(R, C)$ over $N=P \cup Q$ where $R$ is a finite subset of $Q \cdot P \times(T \cup\{\varepsilon\}) \times Q . P^{*}$ and $C$ is a rational subset of $Q . P^{*}$. The closure by isomorphism $[G(R, C)]$ of their transition graphs form the family $R E C_{R a t}$.

Proposition 4.2 We have

$G \in R E C_{\text {Rat }} \Longleftrightarrow G$ isomorphic to $\overline{R . N^{*}} \mid C$ for some system $(R, C)$.

\section{Proof.}

$\Longleftarrow$ : by Theorem 3.6 and Proposition 3.9.

$\Longrightarrow$ : Let $G \in R E C_{\text {Rat }}$. By Theorem 3.7, $G$ is isomorphic to the following graph:

$$
H:=\left(\bigcup_{i=1}^{n}\left(U_{i} \stackrel{a_{i}}{\rightarrow} V_{i}\right) . N^{*}\right)_{\mid L} \text { with } L, U_{1}, V_{1}, \ldots, U_{n}, V_{n} \in \operatorname{Rat}\left(N^{*}\right)
$$

For every $1 \leq i \leq n$, let $\left(G_{i}, r_{i}, E_{i}\right)$ and $\left(H_{i}, s_{i}, F_{i}\right)$ be finite $N$-automata recognizing respectively $U_{i}$ and $V_{i}$. We may assume that $V_{G_{1}}, V_{H_{1}}, \ldots, V_{G_{n}}, V_{H_{n}}$ are pairwise disjoint. Let $\$$ be a new symbol. We define the following rewriting system $(R, C)$ : 


$$
R \mid \begin{array}{rlll}
\$ & \stackrel{a_{i}}{\longrightarrow} & r_{i} & \text { for any } 1 \leq i \leq n \\
p A & \stackrel{\varepsilon}{\longrightarrow} q & \text { for any } p \frac{A}{G_{i}} q \text { with } 1 \leq i \leq n \\
p & \stackrel{\varepsilon}{\longrightarrow} t \quad \text { for any } p \in E_{i}, t \in F_{i} \text { with } 1 \leq i \leq n \\
t & \stackrel{\varepsilon}{\longrightarrow} s A & \text { for any } s \frac{A}{H_{i}} t \text { with } 1 \leq i \leq n \\
s_{i} & \stackrel{\varepsilon}{\longrightarrow} \$ \quad \text { for any } 1 \leq i \leq n
\end{array}
$$

and $C=\$ L$. So $\overline{R . N^{*}}{ }_{\mid C}=\$ . H$.

Another particular labelled rewriting systems are the Turing machines with a read only input tape and a working tape [MS 97], [Pay 00]. More exactly and given an alphabet $Q$ of states, a disjoint alphabet $T$ of input tape letters, and a disjoint alphabet $P_{\square}=P \cup\{\square\}$ of working tape letters, a (non deterministic) labelled Turing machine $(M, C)$ is a finite set $M$ of rules of the form:

$$
p A \stackrel{a}{\longrightarrow} q B \delta \text { where } p, q \in Q, a \in T \cup\{\varepsilon\}, A, B \in P_{\square}, \delta \in\{+,-\}
$$

with a rational set $C \in \operatorname{Rat}\left(\left(Q \cup P_{\square}\right)^{*}\right)$ of configurations.

However we are only interested to configurations upv where $p \in Q$ and $u, v \in P_{\square}{ }^{*}$ with $u(1), v(|v|) \neq \square$. Precisely a configuration is of the form $] u] p[v$ [ where for any word $u \in P_{\square}^{*},[u[$ (resp. $] u]$ ) is the greatest prefix (resp. suffix) of $u$ having its last (resp. first) letter distinct of $\square$ i.e. by induction,

$$
[u \square[=[u[\wedge[u[=u \text { if } u(|u|) \neq \square \text { and }] \square u]=] u] \wedge] u]=u \text { if } u(1) \neq
$$

The set of transitions of $M$ is the following $(T \cup\{\varepsilon\})$-graph:

$$
\begin{aligned}
T(M) & :=\{] u] p[A v[\stackrel{a}{\longrightarrow}] u B] q\left[v\left[\mid p A \underset{M}{\stackrel{a}{\longrightarrow}} q B+\wedge u, v \in P_{\square}^{*}\right\}\right. \\
& \cup\{] u C] p[A v[\stackrel{a}{\longrightarrow}] u] q\left[C B v\left[\mid p A \underset{M}{\stackrel{a}{\longrightarrow}} q B-\wedge C \in P_{\square} \wedge u, v \in P_{\square}{ }^{*}\right\}\right.
\end{aligned}
$$

Hence the transition graph of any labelled Turing machine $(M, C)$ is the T-graph:

$$
G(M, C):=\overline{T(M)}_{\mid C}
$$

The transition graph of any labelled Turing machine is the transition graph of a stable labelled rewriting system $(R, C)$ meaning that $C$ is stable in $T(R)$ :

$$
s \vec{R}^{*} r \vec{R}^{*} t \wedge s, t \in C \Longrightarrow r \in C
$$

Lemma 4.3 We can transform any labelled Turing machine $M$ into a stable labelled rewriting system $(R, C)$ such that $T(R)_{\mid C}$ is isomorphic to $T(M)$.

\section{Proof.}

We take a new symbol $\$$ and the following rational language: 


$$
C=\left\{\$ u p v \$ \mid p \in Q \wedge u, v \in P_{\square}^{*} \wedge u(1), v(|v|) \neq \square\right\}
$$

We transform any rule $p A \stackrel{a}{\longrightarrow} q B+$ of $M$ into the following rules:

$$
\begin{array}{lll}
C p A & \stackrel{a}{\longrightarrow} C B q & \text { if } C B \neq \$ \square \\
C p \$ & \stackrel{a}{\longrightarrow} C B q \$ & \text { if } A=\square \wedge C B \neq \$ \square \\
\$ p A & \stackrel{a}{\longrightarrow} \$ q & \text { if } B=\square \\
\$ p \$ & \stackrel{a}{\longrightarrow} \$ q \$ & \text { if } A=B=\square
\end{array}
$$

We transform any rule $p A \stackrel{a}{\longrightarrow} q B$ - of $M$ into the following rules:

$$
\begin{aligned}
& C p A D \stackrel{a}{\longrightarrow} q C B D \quad \text { if } C \neq \$ \wedge B D \neq \square \$ \\
& C p A \$ \stackrel{a}{\longrightarrow} q C \$ \quad \text { if } B=\square \wedge C \neq \square, \$ \\
& \square p A \$ \stackrel{a}{\longrightarrow} q \$ \quad \text { if } B=\square \\
& C p \$ \stackrel{a}{\longrightarrow} q C B \$ \quad \text { if } A=\square \neq B \wedge C \neq \$ \\
& C p \$ \stackrel{a}{\longrightarrow} q C \$ \quad \text { if } A=B=\square \wedge C \neq \square, \$ \\
& \square p \$ \stackrel{a}{\longrightarrow} q \$ \quad \text { if } A=B=\square \\
& \$ p A D \stackrel{a}{\longrightarrow} \$ q \square B D \quad \text { if } B D \neq \square \$ \\
& \$ p A \$ \stackrel{a}{\longrightarrow} \$ q \$ \quad \text { if } B=\square \\
& \$ p \$ \stackrel{a}{\longrightarrow} \$ q \square B \$ \quad \text { if } A=\square \neq B \\
& \$ p \$ \stackrel{a}{\longrightarrow} \$ q \$ \quad \text { if } A=B=\square
\end{aligned}
$$

In this way, we obtain a labelled rewriting system $R$ such that

$$
\begin{aligned}
C \text { is closed by } \underset{R}{\longrightarrow} \text { hence } & (R, C) \text { is stable } \\
U \underset{T(M)}{\stackrel{a}{\longrightarrow}} V & \Longleftrightarrow \quad \$ U \$ \frac{a}{T(R)} \$ V \$ \wedge \$ U \$, \$ V \$ \in C
\end{aligned}
$$

Thus $\$ T(M) \$=T(R)_{\mid C}$.

Conversely and up to the $\varepsilon$-transitions, any labelled rewriting system can be simulated by a labelled Turing machine.

Lemma 4.4 We can transform any labelled rewriting system $R$ into a labelled Turing machine $(M, C)$ such that $\overline{T(M)}_{\mid C}$ is isomorphic to $\overline{T(R)}$.

\section{Proof.}


We denote by $m_{1}$ (resp. $m_{2}$ ) the maximum length of the left (resp. right) hand sides of the rules of $R$ i.e.

$$
\begin{aligned}
m_{1} & =\max \{|U| \mid \exists a, V,(U, a, V) \in R\} \\
\text { and } m_{2} & =\max \{|V| \mid \exists U, a,(U, a, V) \in R\}
\end{aligned}
$$

We take two new symbols - and $\$$, and we define the following state set $Q$ of the labelled Turing machine to be constructed:

$$
Q=\{\bullet\} \cup N^{\leq m_{1}} \times\left(N \leq m_{2} \cup\{\$\}\right) \times(T \cup\{\varepsilon\})
$$

We take the following set $M^{\prime}$ of Turing rules:

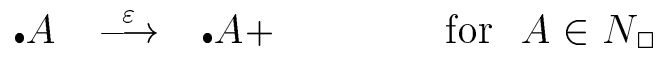

$$
\begin{aligned}
& \text { - } A \stackrel{\varepsilon}{\longrightarrow} \cdot A-\quad \text { for } A \in N_{\square} \\
& \text { - } A \stackrel{\varepsilon}{\longrightarrow}(U, V, a) A+\quad \text { for } \quad A \in N_{\square} \text { and }(U, a, V) \in R \\
& (A U, B V, a) A \stackrel{\varepsilon}{\longrightarrow}(U, V, a) B+ \\
& (\varepsilon, B V, a) A \stackrel{\varepsilon}{\longrightarrow}(\varepsilon, V A, a) B+\quad \text { for } \quad A \in N \\
& (\varepsilon, B V, a) \square \stackrel{\varepsilon}{\longrightarrow}(\varepsilon, V, a) B+ \\
& (A U, \varepsilon, a) A \stackrel{\varepsilon}{\longrightarrow}(U B, \varepsilon, a) B+\text { for } B \in N \\
& (A U, \varepsilon, a) A \stackrel{\varepsilon}{\longrightarrow}(U, \$, a) \square+ \\
& (A U, \$, a) A \stackrel{\varepsilon}{\longrightarrow}(U, \$, a) \square+ \\
& (\varepsilon, \$, a) \square \stackrel{\varepsilon}{\longrightarrow}(\varepsilon, \varepsilon, a) \square+
\end{aligned}
$$

In this way, we obtain a labelled Turing machine $M^{\prime}$ such that for every $a \in T \cup\{\varepsilon\}$ and $U, V \in N^{*}$,

$$
U \underset{T(R)}{\stackrel{a}{\longrightarrow}} V \quad \Longleftrightarrow \quad \cdot U \underset{T\left(M^{\prime}\right)}{\stackrel{\varepsilon}{\rightleftharpoons}} X(\varepsilon, \varepsilon, a) Y \wedge[X Y[=V
$$

We complete $M^{\prime}$ to $M$ by adding the following rules:

$$
(\varepsilon, \varepsilon, a) A \stackrel{a}{\longrightarrow} \cdot A+\quad \text { for } A \in N_{\square}
$$

The relation $h=\left\{(U, \cdot U) \mid U \in N^{*}\right\}$ is a partial weak isomorphism from $T(R)$ into $T(M)$. More precisely and for every $a \in T \cup\{\varepsilon\}$, we have

$$
\begin{aligned}
& U \underset{T(R)}{\stackrel{a}{\longrightarrow}} V \Longrightarrow \quad \cdot U \underset{T(M)}{\stackrel{a}{\Longrightarrow}} \cdot V \\
& \text { •U} \underset{T(M)}{\stackrel{a}{\Rightarrow}} \cdot V \Longrightarrow U \underset{T(R)}{\stackrel{a}{\Rightarrow}} V
\end{aligned}
$$


So $h$ is a partial isomorphism from $\overline{T(R)}$ into $\overline{T(M)}$.

Thus $h$ is a partial isomorphism from $\overline{T(R)}$ into $\overline{T(M)}_{\mid C}$ where $C=\operatorname{Im}(h)=$ - $N^{*}$.

As $V_{\overline{T(R)}} \subseteq N^{*}=\operatorname{Dom}(h)$, the graphs $\overline{T(R)}$ and $\overline{T(M)}_{\mid C}$ are isomorphic.

The rewriting systems and the Turing machines have the same transition graphs.

Theorem 4.5 The labelled Turing machines and the labelled rewriting systems define up to isomorphism, the same family of transition graphs, and their traces are the recursively enumerable languages.

\section{Proof.}

i) Let $(M, D)$ be a labelled Turing machine.

By Lemma 4.3 , we can construct a stable labelled rewriting system $(R, C)$ and an isomorphism $h$ from $T(M)$ to $T(R)_{\mid C}$.

By restriction, $h$ defines an isomorphism from $\overline{T(M)}$ to $\overline{T(R)_{\mid C}}$.

By Equation (2.2), $\overline{T(R)_{\mid C}}={\overline{T(R)_{\mid C}}}_{\mid r}=G(R, C)$. Thus $G(M, D)=\overline{T(M)}_{\mid D}$ is isomorphic (by a restriction of $h$ ) to $\overline{T(R)}_{\mid C \cap h(D)}=G(R, C \cap h(D))$.

ii) Let $(R, C)$ be a labelled rewriting system.

By Lemma 4.4, we can construct a labelled Turing machine $(M, D)$ and an isomorphism $h$ from $\overline{T(R)}$ to $\overline{T(M)}_{\mid D}$. Thus $G(R, C)=\overline{T(R)}_{\mid C}$ is isomorphic (by a restriction of $h)$ to $\overline{T(M)}_{\mid h(C) \cap D}=G(M, h(C) \cap D)$.

We denote by TURING $G_{T}$ the family of $T$-graphs isomorphic to the transition graphs of labelled Turing machines (or of labelled rewriting systems). As for the previous graph families (investigated in the previous section), we characterize the family $T U R I N G_{T}$ by inverse mappings of the binary tree. The images of these mappings can be the class of recursively enumerable languages, or can be only the class of the rational closure $\overline{\operatorname{L}}$ in $(R a t)$ of $\overline{\operatorname{L}}$ in.

Theorem 4.6 We have TURING $G_{T}=R E C_{\overline{L i n}(R a t)_{T}}=R E C_{R E_{T}}$

\section{Proof.}

i) Let us show that $T U R I N G_{T} \subseteq R E C_{\bar{L} i n(R a t)}{ }_{T}$.

Let $(R, C)$ be a labelled rewriting system: $R$ is a finite subset of $N^{*} \times(T \cup\{\varepsilon\}) \times N^{*}$ and $C$ is a rational subset of $N^{*}$.

We replace in $R$ the label $\varepsilon$ by a new letter $\$$ :

$$
S:=\{(u, a, v) \in R \mid a \in T\} \cup\{(u, \$, v) \mid(u, \varepsilon, v) \in R\}
$$

So $T(S)=h^{-1}\left(\Lambda_{N}\right)$ where $h$ is the following linear mapping: 


$$
h(a)=\left\{\tilde{\bar{x}} \widetilde{u} v \mid(u, a, v) \in S \wedge x \in N^{*}\right\} \text { for every } a \in T \cup\{\$\}
$$

Furthermore $\overline{T(R)}=g^{-1}(T(S))$ where $g$ is the following rational mapping:

$$
g(a)=\$^{*} a \$^{*} \text { for every } a \in T
$$

By (2.1), we have $\overline{T(R)}=(g \circ h)^{-1}\left(\Lambda_{N}\right)$ where $g \circ h$ is the following mapping:

$$
(g \circ h)(a)=h(g(a))=h\left(\$^{*} a \$^{*}\right)=h(\$)^{*} h(a) h(\$)^{*} \in \overline{\operatorname{Lin}}(\text { Rat })
$$

Finally and by Proposition 3.5, G(R,C)= $\overline{T(R)}_{\mid C} \in R E C_{\left.\overline{\operatorname{Lin}}_{(\mathrm{Rat})}\right)_{T}}$.

ii) $R E C_{\bar{L} \text { in(Rat) }} \subseteq R E C_{R E}$ because $\bar{L}$ in $(R a t) \subseteq R E$.

iii) Let us show that $R E C_{R E_{T}} \subseteq T U R I N G_{T}$.

Let a mapping $h: T \longrightarrow R E\left(\{a, b, \bar{a}, \bar{b}\}^{*}\right)$. By Proposition 3.5, it is sufficient to construct a rewriting system $(R, C)$ such that $h^{-1}\left(\Lambda_{\{a, b\}}\right)$ is isomorphic to $\overline{T(R)}_{\mid C}$. For every $c \in T$, there is a Turing machine $M_{c}$ : a finite set of rules of the form:

$$
p A \stackrel{x}{\rightarrow} q B \delta \text { where } p, q \in Q_{c}, x \in\{\varepsilon, a, b, \bar{a}, \bar{b}\}, A, B \in P_{c} \cup\{\square\}, \delta \in\{+,-\}
$$

plus an initial configuration $i_{c}$ and a set $F_{c} \subseteq Q_{c}$ of final states recognizing:

$$
h(c)=\mathcal{L}\left(T\left(M_{c}\right), i_{c},\{] u\right] q\left[v\left[\mid q \in F_{c} \wedge u, v \in\left(P_{c} \cup \square\right)^{*}\right\}\right)
$$

Up to renaming, we may assume that the sets $\left(P_{c}\right)_{c \in T},\left(Q_{c}\right)_{c \in T}$ are pairwise disjoints, and we define the following Turing machine:

$$
\begin{aligned}
M & =\left\{p A \stackrel{\varepsilon}{\longrightarrow} q B \delta \in M_{c} \mid c \in T\right\} \\
& \cup\left\{p A \stackrel{\varepsilon}{\longrightarrow} q_{x} B \delta \mid \exists c \in T, p A \stackrel{x}{\longrightarrow} q B \delta \in M_{c} \wedge x \neq \varepsilon\right\}
\end{aligned}
$$

We take three new symbols $\$, \&$, and we construct a rewriting system $R$. First, we take the following rules:

$$
\$ \$ \stackrel{\varepsilon}{\longrightarrow} \$ i_{c} \$ \quad \text { for every } \quad c \in T
$$

to describe the moves between two $\$$ of the Turing machines defining $h$. We transform (as in Lemma 4.3) any rule $p A \stackrel{\varepsilon}{\longrightarrow} q B+$ of $M$ into the following rules:

$$
\begin{array}{|lll}
C p A & \stackrel{\varepsilon}{\longrightarrow} C B q & \text { if } C B \neq \$ \square \\
C p \$ \stackrel{\varepsilon}{\longrightarrow} C B q \$ & \text { if } A=\square \wedge C B \neq \$ \square \\
\$ p A \stackrel{\varepsilon}{\longrightarrow} \$ q & \text { if } B=\square \\
\$ p \$ \stackrel{\varepsilon}{\longrightarrow} \$ q \$ & \text { if } A=B=\square
\end{array}
$$

In a same way (and as in the proof of Lemma 4.3), we transform any rule $p A \stackrel{\varepsilon}{\longrightarrow} q B$ of $M$ into new rules of $R$.

For every $c \in T, q \in Q_{c}, A \in P_{c} \cup\{\square\}, y \in\{a, b, \bar{a}, \bar{b}\}, x \in\{a, b\}$, we take the rules: 


$$
\begin{array}{rll}
q_{y} & \stackrel{\varepsilon}{\longrightarrow} & q_{y}^{\prime} \& \\
A q_{y}^{\prime} & \stackrel{\varepsilon}{\longrightarrow} & q_{y}^{\prime} A \\
x \$ q_{\bar{x}}^{\prime} & \stackrel{\varepsilon}{\longrightarrow} & \$ \bar{q} \\
\$ q_{x}^{\prime} & \stackrel{\varepsilon}{\longrightarrow} & x \$ \bar{q} \\
\bar{q} A & \stackrel{\varepsilon}{\longrightarrow} & A \bar{q} \\
\bar{q} \& & \stackrel{\varepsilon}{\longrightarrow} & q
\end{array}
$$

For the acceptance and for every $c \in T$ and $A \in\left(\cup_{c} P_{c}\right) \cup\{\square\}$, we define

$$
\begin{array}{|lll}
q & \stackrel{c}{\longrightarrow} \text {. if } q \in F_{c} \\
. A & \stackrel{\varepsilon}{\longrightarrow} \cdot \\
A . & \stackrel{\varepsilon}{\longrightarrow} \cdot \\
\$ . \$ & \stackrel{\varepsilon}{\longrightarrow} \$ \$
\end{array}
$$

For every $c \in T$ and $u, v \in\{a, b\}^{*}$, we have

$$
u \underset{h^{-1}\left(\Lambda_{\{a, b\}}\right)}{\stackrel{c}{\longrightarrow}} v \Longleftrightarrow u \$ \underset{T(R)}{\stackrel{c}{\rightleftharpoons}} v \$ \$
$$

So $h=\left\{(u, u \$ \$) \mid u \in\{a, b\}^{*}\right\}$ is a partial weak isomorphism from $h^{-1}\left(\Lambda_{\{a, b\}}\right)$ into $T(R)$. Thus $h$ is a partial isomorphism from $\overline{h^{-1}\left(\underline{\left.\Lambda_{\{a, b\}}\right)}\right.}=h^{-1}\left(\Lambda_{\{a, b\}}\right)$ into $\overline{T(R)}$. Hence $h$ is an isomorphism from $h^{-1}\left(\Lambda_{\{a, b\}}\right)$ into $\overline{T(R)_{\mid C}}$ where $C=\operatorname{Im}(h)=$ $\{a, b\} * \$$.

The class $T U R I N G$ is the closure of $R A T$ by inverse rational mapping.

Theorem 4.7 We have $\operatorname{Rat}_{T}^{-1}\left(R A T_{N}\right)=T U R I N G_{T}$.

\section{Proof.}

i) $T U R I N G_{T} \subseteq \operatorname{Rat}_{T}^{-1}\left(R A T_{N}\right)$.

Let $G \in T U R I N G_{T}: G$ is isomorphic to $\overline{T(R)}_{\mid C}$ for some labelled rewriting system $(R, C)$. Let $\#, \$$ be two new symbols. We have

$$
\overline{T(R)}=h^{-1}(T(S))
$$

where $h$ is the rational mapping defined by $h(a)=\$^{*} a \$^{*}$ for every $a \in T$, and $S$ is the system obtained from $R$ by replacing the label $\varepsilon$ by $\$$ :

$$
S:=\{(u, a, v) \in R \mid a \in T\} \cup\{(u, \$, v) \mid(u, \varepsilon, v) \in R\}
$$

By Equations (2.4) and (2.1), we have 


$$
\overline{T(R)}_{\mid C}=h_{\#}^{-1}(T(S) \cup\{u \stackrel{\#}{\longrightarrow} u \mid u \in C\})
$$

where $h_{\#}(a)=\# \$^{*} a \$^{*} \#$ for every $a \in T$.

Obviously $T(S)$ is a rational graph and $\{u \stackrel{\#}{\longrightarrow} u \mid u \in C\}$ is also a rational graph because $C$ is a rational language. Hence $G \in \operatorname{Rat}_{T}^{-1}\left(R A T_{N}\right)$.

ii) $\operatorname{Rat}_{T}^{-1}\left(R A T_{N}\right) \subseteq T U R I N G_{T}$.

Let $G \in \operatorname{Rat}_{T}^{-1}\left(R A T_{N}\right)$ : there is a rational $N$-graph $H$ and a mapping $h: T \longrightarrow \operatorname{Rat}\left(N^{*}\right)$ such that $G=h^{-1}(H)$.

By definition of a rational graph, there is an alphabet $X$ and a $N$-labelled transducer $A=\left(K, i,\left(E_{x}\right)_{x \in N}\right)$ where $K$ is a finite $\left(X^{*} \times X^{*}\right)$-automaton, $i$ is the initial state, and for each $x \in N, E_{x}$ is a set of final states, and such that the automaton $A$ recognizes the graph $\mathcal{L}(A)=\left\{u \stackrel{x}{\longrightarrow} v \mid \exists s \in E_{x}, i \underset{K}{\stackrel{u / v}{\longrightarrow}} s\right\}$ which is isomorphic to $H$.

Furthermore and for each $a \in T$, there is a finite $N$-automaton $\left(K_{a}, i_{a}, F_{a}\right)$ recognizing the rational language $h(a)$.

We may assume that the automata $\left(K_{a}\right)_{a \in T}$ have pairwise disjoint state sets: $V_{K_{a}} \cap$ $V_{K_{b}}=\emptyset$ for $a \neq b$. We denote by $\bar{K}=\bigcup_{a \in T} K_{a}$ and we take a new state $\bar{\imath} \notin V_{\bar{K}}$.

We take a new symbol $\$$ and we denote by $C=\$ \bar{\imath} X^{*} \$$ the (rational) configuration set of the following rewriting system $R$ :

$$
\begin{aligned}
& R \mid \begin{array}{rlll}
\bar{\imath} & \stackrel{\varepsilon}{\longrightarrow} i_{a} & \text { for each } a \in T \\
\$ s & \stackrel{\varepsilon}{\longrightarrow} \$(i, s) & \text { for each } s \in V_{\bar{K}} \\
\$ s & \stackrel{a}{\longrightarrow} \$ \bar{\imath} & \text { if } s \in F_{a} \\
(p, s) u & \stackrel{\varepsilon}{\longrightarrow} v(q, s) & \text { if } p \stackrel{u / v}{\longrightarrow} q \text { and } s \in V_{\bar{K}} \\
(p, s) \$ & \stackrel{\varepsilon}{\longrightarrow} t \$ & \text { if } p \in E_{x} \text { and } s \frac{x}{\bar{K}} t \text { for some } x \in N \\
A s & \stackrel{\varepsilon}{\longrightarrow} s A & \text { for each } A \in X \text { and } s \in V_{\bar{K}}
\end{array} \\
& \overline{T(R)}_{\mid C}=\{\$ \bar{\imath} u \$ \stackrel{a}{\rightarrow} \$ \bar{\imath} v \$ \mid \$ \bar{\imath} u \$ \underset{T(R)}{\stackrel{a}{\longrightarrow}} \$ \bar{\imath} v \$\} \\
& =\left\{\$ \bar{\imath} u \$ \stackrel{a}{\longrightarrow} \$ \bar{\imath} v \$ \mid \exists s \in F_{a} \quad \$ i_{a} u \$ \underset{T(R)}{\stackrel{\varepsilon}{\longrightarrow}} \$ s v \$\right\} \\
& =\left\{\$ \bar{\imath} u \$ \stackrel{a}{\longrightarrow} \$ \bar{\imath} v \$ \mid \exists w \in h(a) u \frac{w(1)}{L(A)} \ldots \frac{w(|w|)}{L(A)} v\right\}
\end{aligned}
$$

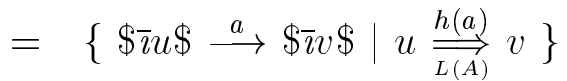

$$
\begin{aligned}
& =\$ \bar{\imath} h^{-1}(L(A)) \$ \text { isomorphic to } h^{-1}(H)=G \text {. }
\end{aligned}
$$

In particular $R A T$ is not closed by inverse rational mapping. We also deduce that the transition graphs of labelled Turing machines are the rational restrictions of the 
$\varepsilon$-closure of rational graphs (with $\varepsilon$-arcs).

An equivalent way to get the family $T U R I N G_{T}$ is to consider the computable relations of single tape non deterministic Turing machines. Precisely and given an alphabet $Q$ of states and a disjoint alphabet $P_{\square}=P \cup\{\square\}$ of working tape letters, a (single tape non deterministic) Turing machine $M$ is a finite set of rules of the form:

$$
p A \longrightarrow q B \delta \text { where } p, q \in Q, A, B \in P_{\square}, \delta \in\{+,-\}
$$

The set of transitions of $M$ is the previous graph $T(M)$ which is unlabelled. For a $T$-labelling and as for the labelled transducers recognizing the rational graphs, we take a subset $F_{a} \subseteq Q$ of final states for each letter $a \in T$. Furthermore and as usual, we take an initial state $q_{0} \in Q$. In this way, a Turing machine $M$ defines the following T-computation graph:

$$
R(M):=\left\{u \stackrel{a}{\longrightarrow} \overleftarrow{v} \vec{w} \mid u \in P^{*} \wedge a \in T \wedge \exists q \in F_{a} \quad p_{0} u \underset{T(M)}{\Longrightarrow} v q w\right\}
$$

where $\overleftarrow{v}$ is the greatest suffix in $P^{*}$ of $v$, and $\vec{w}$ is the greatest prefix in $P^{*}$ of $w$ The transition graphs of labelled Turing machines are the computation graphs of unlabelled Turing machines.

Theorem 4.8 The family TURING $G_{T}$ is the set of T-graphs isomorphic to the computation graphs of Turing machines.

\section{Proof.}

$\subseteq$ : Let $(M, C)$ be a labelled Turing machine.

Let $T$ be its label alphabet, $Q$ be its state alphabet and $P$ be its tape set.

We have to construct a (unlabelled non deterministic) Turing machine $N$ such that its computable graph $R(N)$ is isomorphic to $G(M, C)$.

Such an isomorphism is given by the mapping which associates to any configuration $u p v$ where $p \in Q$ and $u, v \in P_{\square}^{*}$ with $u(1), u(|u|) \neq \square$, the word \& $\bar{u} \bar{p} \bar{v} \$$ with $\bar{p}$ in a new alphabet $\bar{Q}$ in bijection with $Q, \bar{u}$ (resp. $\bar{v}$ ) are obtained from $u$ (resp. $v$ ) by replacing $\square$ by a new symbol $\bar{\square}$, and \&, $\$$ are also new symbols.

So we have to construct a Turing machine $N$ such that

$$
u \underset{G(M, C)}{\stackrel{a}{\longrightarrow}} v \Longleftrightarrow \& \bar{u} \$ \underset{R(N)}{\longrightarrow} \& \bar{v} \$
$$

For $p_{0}$ the initial state of $N$ and $f_{a}$ the unique final state for each label $a \in T$, we will construct $N$ in such a way that

$$
u \underset{T(M)}{\stackrel{\varepsilon}{\rightleftharpoons}} \frac{a}{T(M)} \underset{T(M)}{\stackrel{\varepsilon}{\rightleftharpoons}} v \quad \Longleftrightarrow \quad p_{0} \& \bar{u} \$ \underset{T(N)}{\Longrightarrow} \& \bar{v} f_{a} \$ \quad \text { for any } u, v \in C
$$

As $C$ is a rational set of configurations, there is a finite $\left(Q \cup P_{\square}\right)$-automaton $(G, i, F)$ recognizing $C: \mathcal{L}(G, i, F)=C$.

First, the Turing machine $N$ checks that the input word (between \& and $\$$ ) belongs 
to $C$ :

$$
\begin{aligned}
p_{0} \& & \longrightarrow i \&+ \\
s A & \rightarrow t A+\quad \text { if } s \frac{A}{G} t \text { and } A \in P \\
s \bar{\square} \rightarrow t \square+ & \text { if } s \frac{\square}{G} t \\
s \bar{p} \longrightarrow t \bar{p}+ & \text { if } s \frac{p}{G} t \text { and } p \in Q \\
s \$ \rightarrow \hat{\square}- & \text { if } s \in F \\
\hat{A} B \longrightarrow \hat{B} A- & \text { if } A \in P_{\square} \cup\{\$\} \text { and } B \in P_{\square} \\
\hat{A} \bar{p} \longrightarrow p A & \text { if } A \in P_{\square} \cup\{\$\} \text { and } p \in Q
\end{aligned}
$$

The last rule without + and - means that we do not move the tape head. Now the machine $N$ simulates any path $\stackrel{a}{\Longrightarrow}$ of $M$ :

$$
\begin{aligned}
& p A \longrightarrow q B \delta \quad \text { if } p A \stackrel{\varepsilon}{\longrightarrow} q B \delta \text { is a rule of } M \\
& p A \longrightarrow q_{a} B \delta \quad \text { if } p A \stackrel{a}{\longrightarrow} q B \delta \text { is a rule of } M \\
& p_{a} A \longrightarrow q_{a} B \delta \text { if } p A \stackrel{\varepsilon}{\longrightarrow} q B \delta \text { is a rule of } M
\end{aligned}
$$

Furthermore $N$ must push the endmarkers \& and $\$$ when they are accessible: for any $p \in Q \cup \bigcup_{a \in T} Q_{a}$

$$
\begin{aligned}
p \$ & \longrightarrow p^{\prime} \square+ \\
p^{\prime} \square & \longrightarrow p \$- \\
p \& & \longrightarrow p^{\prime \prime} \square- \\
p^{\prime \prime} \square & \longrightarrow p \&+
\end{aligned}
$$

Then $N$ removes the useless $\square$ (on the right of \& and on the left of $\$$ ) and add the label $a \in T$ after the right endmarker $\$$ : 


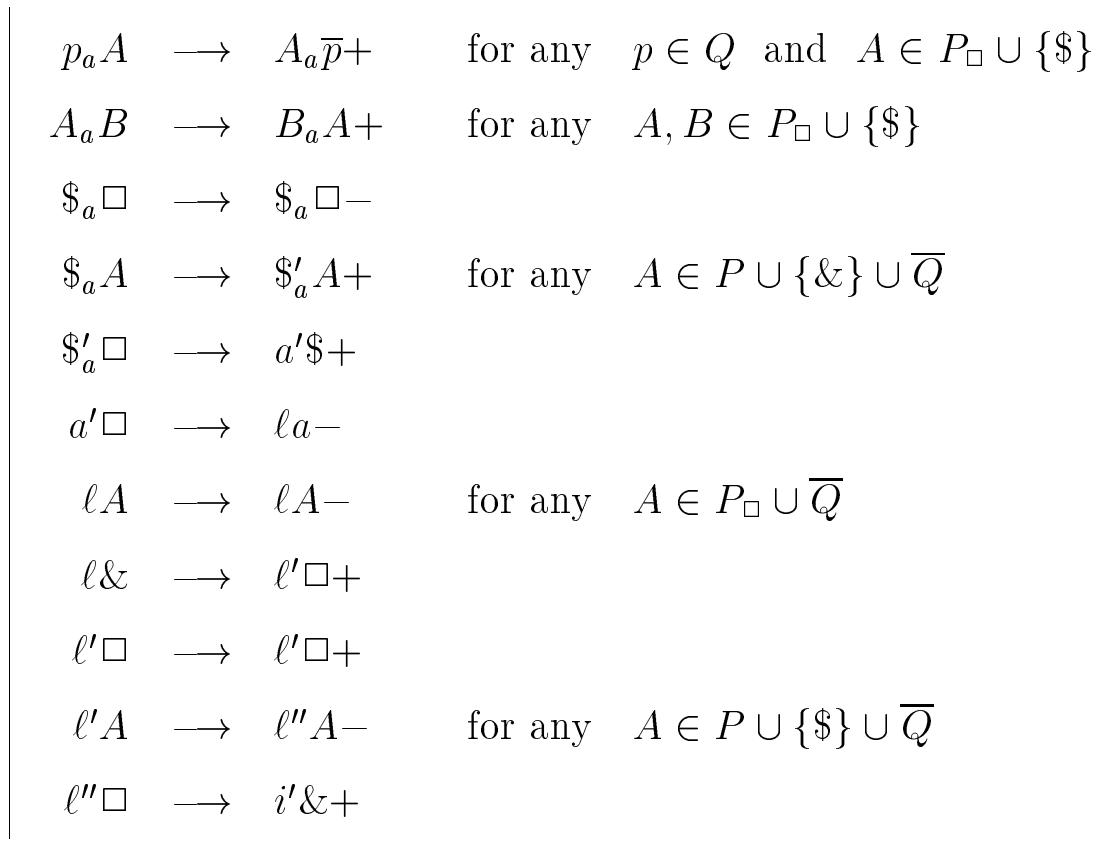

At this step, $N$ reaches a configuration of the form \& $v \bar{p} w \$ a$ with $v(1), w(|w|) \neq \square$ with a state $i^{\prime}(i$ is the initial state of the finite automaton $G$ recognizing $C$ ) reading the first letter of $v \bar{p}$. It remains to test whether $v w \in C$, to replace $\square$ by $\bar{\square}$, to remove $a$ and to reach $f_{a}$ :

$$
\mid \begin{array}{lll}
s^{\prime} A & \longrightarrow t^{\prime} A+ & \text { if } s \underset{G}{G} t \text { and } A \in P \cup \bar{Q} \\
s^{\prime} \square & \longrightarrow t^{\prime} \bar{\square}+ & \text { if } s \frac{\square}{G} t \\
s^{\prime} \$ \longrightarrow s^{\prime} \$+ & \text { if } s \in F \\
s^{\prime} a \longrightarrow f_{a} \square- & \text { if } s \in F \text { and } a \in T
\end{array}
$$

$\supseteq$ : Let $N$ be a (unlabelled non deterministic) Turing machine.

Let $P$ be its tape alphabet and $p_{0}$ be its initial state.

We have to construct a labelled Turing machine $(M, C)$ such that its transition graph $G(M, C)$ is isomorphic to the computable graph $R(N)$ of $N$.

We take the rational language $C=i \& P^{*} \$$ where $i, \&, \$$ are new symbols. For the isomorphism, we take the bijection which associates to any $u \in P^{*}$ the word i\&u$u \in C$. So we have to construct a labelled Turing machine $M$ such that

$$
u \underset{R(N)}{\stackrel{a}{\longrightarrow}} v \Longleftrightarrow \quad \Longleftrightarrow \quad u \quad \underset{G(M, C)}{\stackrel{a}{\longrightarrow}} i \& v \$
$$

or equivalently for any $u, v, w \in P^{*}$,

$$
\begin{aligned}
& p_{0} u \underset{T(N)}{\Longrightarrow} \text { xvqwy for some } q \in F_{a}, x \in\left(P^{*} \square\right)^{*} y \in\left(\square P^{*}\right)^{*} \\
& \Longleftrightarrow \quad i \& u \$ \underset{T(M)}{\stackrel{\varepsilon}{T(M)}} \frac{a}{\stackrel{\varepsilon}{T(M)}} i \& v w \$
\end{aligned}
$$

First, the machine $M$ simulates $N$ : 


$$
\begin{aligned}
& i \& \stackrel{\varepsilon}{\longrightarrow} p_{0} \&+ \\
& p A \stackrel{\varepsilon}{\longrightarrow} q B \delta \quad \text { if } p A \longrightarrow q B \delta \text { is a rule of } N \\
& p \$ \stackrel{\varepsilon}{\longrightarrow} p^{\prime} \square+ \\
& p^{\prime} \square \stackrel{\varepsilon}{\longrightarrow} p \$- \\
& p \& \stackrel{\varepsilon}{\longrightarrow} p^{\prime \prime} \square- \\
& p^{\prime \prime} \square \stackrel{\varepsilon}{\longrightarrow} p \&+
\end{aligned}
$$

Then $M$ does a transition by $a$ when $N$ has reached a final state for $a$. And $M$ removes the useless right part (beginning by a $\square$ ) of its configuration:

$$
\begin{aligned}
& p A \stackrel{a}{\rightarrow} \ell A+\quad \text { if } p \in F_{a} \text { and } A \in P \\
& p \$ \stackrel{a}{\longrightarrow} m \$-\quad \text { if } p \in F_{a} \\
& p \square \quad \stackrel{a}{\longrightarrow} j \square+\quad \text { if } p \in F_{a} \\
& \ell A \stackrel{\varepsilon}{\longrightarrow} \ell A+\quad \text { if } A \in P \\
& \ell \$ \stackrel{\varepsilon}{\longrightarrow} m \$- \\
& \ell \square \quad \stackrel{\varepsilon}{\longrightarrow} j \square+ \\
& j A \stackrel{\varepsilon}{\longrightarrow} j \square+\quad \text { if } \quad A \in P_{\square} \\
& j \$ \stackrel{\varepsilon}{\longrightarrow} k \square- \\
& k \square \quad \stackrel{\varepsilon}{\longrightarrow} k \square- \\
& k A \stackrel{\varepsilon}{\longrightarrow} m^{\prime} A+\quad \text { if } \quad A \in P \cup\{\&\} \\
& m^{\prime} \square \stackrel{\varepsilon}{\longrightarrow} m \$-
\end{aligned}
$$

Finally $M$ removes the useless left part (ending by a $\square$ ) of its configuration, and reads the marker \& at state $i$. We do not give this simple part which is similar to the previous one.

As for languages, the family of computation graphs does not change if we restrict to deterministic Turing machines (the set of rules is functional: there is no two rules with the same left hand side).

Theorem 4.9 The family TURING $G_{T}$ is the set of T-graphs isomorphic to the computation graphs of deterministic Turing machines. 
The transformation of a (non deterministic) Turing machine to a deterministic Turing machine with the same computation graph is similar to the usual transformation preserving the recognized language.

\section{Conclusion}

We have presented a hierarchy of graph families and essentially the family $T U R I N G$ of transition graphs of labelled Turing machines with $\varepsilon$-rules. In particular $R E C_{\text {Rat }}$ is the family of transition graphs of pushdown automata with $\varepsilon$-rules. Between the lowest family FIN of finite graphs and the greatest family $T U R I N G$, we can show that the two families $R E C_{R a t}$ and $R A T$ are natural, by considering the Cayley graphs of the word rewriting systems [CK 98], [Ca 00] and [CaK 02]. Finally and using traces, the hierarchy $F I N, R E C_{R a t}, R A T, T U R I N G$ yields a Chomsky hierarchy. Another point is to find a subclass of word rewriting systems such that their transition graphs are the graphs of $R E C_{\text {Lin }}$.

\section{References}

[AN 82] A. ARnold and M. Nivat Comportements de processus, Colloque AFCET 'Les mathématiques de l'informatique', 35-68 (1982).

[Ba 98] K. BARTHELMANn When can an equational simple graph be generated by hyperedge replacement, $23^{\text {rd }}$ MFCS, LNCS 1450, L. Brim, J. Gruska, J. Zlatuska (Eds.), 543-552 (1998).

[Ben 69] M. Benois Parties rationnelles du groupe libre, C.R. Académie des Sciences, Paris, Série A 269, 1188-1190 (1969).

[Ber 79] J. BERSTEL Transductions and context-free languages, Teubner (Ed.), Stuttgart (1979).

[BG 00] A. Blumensath and E. GRÄDEL Automatic structures, $15^{\text {th }}$ LICS, 51-62 (2000).

[BO 93] R. Bоok and F. Отто String-rewriting systems, Texts and monographs in computer science, P. Gries (Ed.), Springer-Verlag (1993).

[Bü 64] R. Büchi Regular canonical systems, Archiv für Mathematische Logik und Grundlagenforschung 6, 91-111 (1964) [reprinted in The collected works of J. Richard Büchi, S. Mac Lane, D. Siefkes (Eds.), Springer-Verlag, New York, 317337 (1990)].

[CK 98] H. CALBRIX and T. KNAPIK A string-rewriting characterization of Muller and Schupp's context-free graphs, $18^{\text {th }}$ FSTTCS, LNCS 1530, V. Arvind, R. Ramanujam (Eds.), 331-342 (1998). 
[Ca 90] D. CAUCAL On the regular structure of prefix rewriting, $15^{\text {th }} \mathrm{CAAP}$, LNCS 431, A. Arnold (Ed.), 87-102 (1990) [a full version is in Theoretical Computer Science 106, 61-86 (1992)].

[Ca 95] D. CAUCAL Bisimulation of context-free grammars and of pushdown automata, CSLI volume 53 "Modal logic and process algebra", A. Ponse, M. de Rijke, Y. Venema (Eds.), Stanford, 85-106 (1995).

[Ca 96] D. CAUCAL On infinite transition graphs having a decidable monadic theory, $23^{\text {rd }}$ ICALP, LNCS 1099, F. Meyer auf der Heide, B. Monien (Eds.), 194-205 (1996) [a full version will appear in Theoretical Computer Science].

[Ca 00] D. CAUCAL On word rewriting systems having a rational derivation, $3^{\text {rd }}$ FOSSACS, LNCS 1784, J. Tiuryn (Ed.), 48-62 (2000).

[CaK 01] D. CAUCAL and T. KNAPIK An internal presentation of regular graphs by prefix-recognizable ones, Theory of Computing Systems 34-4 (2001).

[CaK 02] D. CAuCAL and T. KNAPIK On a Chomsky-like hierarchy of infinite graphs, $27^{\text {th }}$ MFCS, K. Diks, W. Rytter (Eds.), to appear in LNCS (2002).

[Co 90] B. Courcelle Graph rewriting: an algebraic and logic approach, Handbook of Theoretical Computer Science Vol. B, J. Leeuwen (Ed.), Elsevier, 193-242 (1990).

[DJ 90] N. Dershowitz and J.-P. JouAnnaud Rewrite systems, Handbook of Theoretical Computer Science Vol. B, J. Leeuwen (Ed.), Elsevier, 243-320 (1990).

[EM 65] C. Elgot and J. Mezei On relations defined by generalized finite automata, IBM J. Res. Dev. 9, 47-68 (1965).

[FS 93] C. FRougny and J. SAKARovitCH Synchronized rational relations of finite and infinite words, Theoretical Computer Science 108, 45-82 (1993).

[MS 97] A. Mateescu and A. SalomaA Aspects of classical language theory, Handbook of Formal Languages Vol. 1, G. Rozenberg, A. Salomaa (Eds.), SpringerVerlag, 175-251 (1997).

[MuS 85] D. Muller and P. Schupp The theory of ends, pushdown automata, and second-order logic, TCS 37, 51-75 (1985).

[Mo 00] C. Morvan On rational graphs, $3^{\text {rd }}$ FOSSACS, LNCS 1784, J. Tiuryn (Ed.), 252-266 (2000).

[MoS 01] C. Morvan and C. STIRling Rational graphs trace context-sensitive languages, $26^{\text {th }}$ MFCS, P. Kolman, A. Pultr and J. Sgall (Eds.), LNCS 2136, 548559 (2001).

[MS 85] D. Muller and P. Schupp The theory of ends, pushdown automata, and second-order logic, Theoretical Computer Science 37, 51-75 (1985).

[Pay 00] E. PAYET Produit synchronisé pour quelques classes de graphes infinis, PhD Thesis, University of La Réunion (2000).

[Ri 01] C. RISPAL The synchronized graphs trace the context-sensitive languages, DEA report, University of Rennes (2001).

[Ur 00] T. URVOY Regularity of congruential graphs, 25 $5^{\text {th }}$ MFCS, LNCS 1893, M. Nielsen and B. Rovan (Eds.), 680-689 (2000). 